H. Tamura

Nagoya Math. J.

Vol. 89 (1983), 13-45

\title{
THE PRINCIPLE OF LIMITING ABSORPTION AND DECAY \\ OF LOCAL ENERGY FOR THE LINEARIZED EQUATION OF MAGNETOGASDYNAMICS
}

\author{
HIDEO TAMURA
}

\section{§ 0. Introduction}

The present paper is a continuation of [6] and [7] in which the principle of limiting absorption has been verified for symmetric systems of first order with long-range perturbations, but the operators considered there are of constant rank. On the other hand, operators with non-constant rank are also important in application as well as from a purely theoretical point of view. In this paper, we consider the linearized equation of magnetogasdynamics with long-range perturbation as an important example of such operators.

There are only a few works on the spectral and scattering theory for symmetric hyperbolic systems with non-constant rank.

In [5], Ralston considered an operator of the form

$$
L=\sum_{j=1}^{n} A_{j}(x) \partial_{j}+B(x), \quad \partial_{j}=\partial / \partial x_{j},
$$

where he assumed that the symmetric matrices $A_{j}(x)$ are smooth functions and take the constant values $A_{j}^{0}$ for $|x|>R$ and that $B(x)$ is also a smooth function and it vanishes for $|x|>R$. He further assumed that the null space of $A(x, \xi)=\sum_{j=1}^{n} A_{j}(x) \xi_{j}$ is at most one dimensional for $\xi \neq 0$. However, his results do not cover the linearized equation of magnetogasdynamics, since the null space of this system is not one dimensional, as shown later.

Recently, in [2] (or [1]), Avila and Costa considered an operator of the form

$$
L=E(x)^{-1} \sum_{j=1}^{n} A_{j}^{0} \partial_{j},
$$

Received October 13, 1980.

Revised December 23, 1981. 
$A_{j}^{0}$ being a constant symmetric matrix, where they assumed that $E(x)-$ $E_{0}=O\left(|x|^{-\delta}\right), \delta>1$, as $|x| \rightarrow \infty$, for some positive definite matrix $E_{0}$ (shortrange perturbation). Without any assumptions on the roots $\lambda=\lambda(\xi)$ of the characteristic equation

$$
P(\lambda, \xi)=\operatorname{det}\left(\lambda E_{0}-\sum_{j=1}^{n} A_{j}^{0} \xi_{j}\right)=0,
$$

they proved the existence of wave operators associated with $L$ and the unperturbed operator $L_{0}=E_{0}^{-1} \sum_{j=1}^{n} A_{j}^{0} \partial_{j}$. (The completeness was not proved.) Murata [8] has also proved the existence of wave operators for more general symmetric hyperbolic systems.

The perturbed linearized operator of magnetogasdynamics to be discussed here is formulated in the form like (0.2). Under the assumption $E(x)-E_{0}=O\left(|x|^{-\delta}\right), \delta>0$, (long-range perturbation), we shall prove the principle of limiting absorption and, as a result, the decay of local energy is verified for solutions to the symmetric hyperbolic equation $(\partial / \partial t-L) u$ $=0$. (We require some additional assumptions on the derivatives of $E(x)$. The precise formulation is given in Section 1.) In the case of short range perturbations, the completeness of wave operators will be proved by use of the principle of limiting absorption and the detailed result will be stated in another paper.

\section{$\S 1$. Formulation of results}

In this section, we give the precise formulation of the results to be obtained here together with several notations and assumptions.

1.1. Notations. We first list up the notations to be used throughout our entire discussion.

(1) We work exclusively in 3-dimensional euclidean space $R_{x}^{3}$ with generic point $x=\left(x_{1}, x_{2}, x_{3}\right) . \quad R_{\xi}^{3}$ denotes the 3 -dimensional space dual to $R_{x}^{3}$ and the generic point $\xi$ in $R_{\xi}^{3}$ is denoted by $\xi=\left(\xi_{1}, \xi_{2}, \xi_{3}\right)$. Furthermore, we denote by $x \cdot \xi$ the scalar product; $x \cdot \xi=\sum_{j=1}^{3} x_{j} \xi_{j}$.

(2) $C^{k}$ denotes the $k$-dimensional unitary space with the usual scalar product $(()$,$) (The notation (()$,$) is used only for k=3,7$.)

(3) For a multi-index $m=\left(m_{1}, m_{2}, m_{3}\right), m_{j}$ being a non-negative integer, we denote by $|m|$ the length of $m$. We write $\partial_{x}=\left(\partial / \partial x_{1}, \partial / \partial x_{2}, \partial / \partial x_{3}\right), D_{x}$ $=\left(D_{1}, D_{2}, D_{3}\right), D_{j}=-i \partial / \partial x_{j}(i=\sqrt{-1})$ and $\partial_{x}^{m}=\left(\partial / \partial x_{1}\right)^{m_{1}}\left(\partial / \partial x_{2}\right)^{m_{2}}\left(\partial / \partial x_{3}\right)^{m_{3}}$ for 
$m=\left(m_{1}, m_{2}, m_{3}\right)$. (We use the symbols $m$ and $n$ to denote multi-indices throughout this paper.)

(4) We denote by $I_{j}$ the identity matrix of size $j \times j$.

1.2. Functional spaces. We shall introduce the various functional spaces in which we work.

(1) We denote by $H_{s}\left(H_{0}=L_{2}\right)$ the usual Sobolev space of order $s$ over the whole space $R_{x}^{3}$, s being not necessarily a non-negative integer, and the norm in $H_{s}$ is denoted by \|\|$_{s}\left(\|\|_{s}=\mid\left(\begin{array}{l}(1) \\ s, 0\end{array}\right)\right.$.

(2) We denote by $H_{s, \alpha}$ the weighted Sobolev space with weight $\alpha$; $H_{s, \alpha}=\left\{\phi ;\left(1+|x|^{2}\right)^{\alpha / 2} \phi \in H_{s}\right\}$, and the norm in $H_{s, \alpha}$ by $\|\phi\|_{s, \alpha}=\left\|\left(1+|x|^{2}\right)^{\alpha / 2} \dot{\phi}\right\|_{s}$. When $s=0$, we write $H_{0, \alpha}=L_{2, \alpha}$.

(3) We further denote by $H_{s, \alpha}^{(\ell)}$ the direct sum of $H_{s, \alpha}$ ( $\ell$ summands); $H_{s, \alpha}^{(\ell)}=\sum \oplus H_{s, \alpha}\left(L_{2, \alpha}^{(\ell)}=H_{0, \alpha}^{(\ell)}\right)$, and the norm in $H_{s, \alpha}^{(\ell)}$ is denoted by $\left.\right|_{\mid s, \alpha^{*}} ^{(\ell)}$ In the later argument, the space $H_{s, \alpha}^{(7)}(\ell=7)$ is most frequently used, so we simply write ||$_{s, \alpha}$ instead of ||$_{s, \alpha}^{(7)}$ for the norm in $H_{s, \alpha^{*}}^{(7)}$

(4) For a domain $\Omega$ in $R_{x}^{3}$, we denote by $H_{s}(\Omega)$ the Sobolev space of order $s$ over $\Omega$. The notation $H_{s, \alpha}^{(\ell)}(\Omega)$ is also used with the same meaning as above.

1.3. The linearized equation of magnetogasdynamics. The perturbed linearized equation of magnetogasdynamics to be discussed here is described as follows:

$$
\begin{aligned}
& \mu_{0}(\partial / \partial t) h+\nabla \times\left(B_{0} \times v\right)=0, \\
& \rho(x)(\partial / \partial t) v+\nabla p+B_{0} \times(\nabla \times h)=0, \\
& a(x)(\partial / \partial t) p+\nabla \cdot v=0 .
\end{aligned}
$$

Here the unknown functions $h, v$ and $p$ denote the magnetic vector field, velocity vector, and pressure, respectively ( $h$ and $v$ are 3 -dimensional vector-valued functions, $p$ scalar function), while the constants $\mu_{0}$ and $B_{0}$ denote the magnetic permeability and given uniform magnetic vector field, respectively, and $\rho(x)$ and $a(x)$ are given positive scalar functions. The physical meanings of $\rho(x)$ and $a(x)$ will be clarified in the derivation of equations (1.1) $\sim(1.3)$ which is done in Appendix.

We set $u={ }^{t}(h, v, p)$ (7-dimensional vector-valued function). Then, equations $(1.1) \sim(1.3)$ can be put into the symmetric hyperbolic system of the following form:

$$
i(\partial / \partial t) u=E(x)^{-1} L_{0}\left(D_{x}\right) u,
$$


where

$$
\begin{aligned}
& E(x)=\left(\begin{array}{ccc}
\mu_{0} I_{3}, & 0 \\
& \rho(x) I_{3}, & \\
0, & & a(x)
\end{array}\right) \\
& L_{0}\left(D_{x}\right)=\left(\begin{array}{ccc}
0, & \Lambda\left(D_{x}\right)\left[B_{0}\right], & 0 \\
{\left[B_{0}\right] \Lambda\left(D_{x}\right),} & 0, & A\left(D_{x}\right) \\
0, & A^{*}\left(D_{x}\right), & 0
\end{array}\right) \\
& \Lambda\left(D_{x}\right)(=-i \nabla \times)=\left(\begin{array}{ccc}
0, & -D_{3}, & D_{2} \\
D_{3}, & 0, & -D_{1} \\
-D_{2}, & D_{1}, & 0
\end{array}\right), \quad\left(\Lambda^{*}=-\Lambda\right), \\
& A\left(D_{x}\right)(=-i \nabla)={ }^{t}\left(D_{1}, D_{2}, D_{3}\right), \quad A^{*}\left(D_{x}\right)(=-i \nabla \cdot)=\left(D_{1}, D_{2}, D_{3}\right),
\end{aligned}
$$

and $\left[B_{0}\right]$ denotes the $3 \times 3$ matrix corresponding to the operation $B_{0} \times$; $\left[B_{0}\right]^{*}=-\left[B_{0}\right]$.

Without loss of generality, we assume that $B_{0}={ }^{t}(1,0,0)$, so that

$$
\left[B_{0}\right]=\left(\begin{array}{rrr}
0, & 0, & 0 \\
0, & 0, & -1 \\
0, & 1, & 0
\end{array}\right) \text {. }
$$

We further assume that $\rho(x)$ and $a(x)$ converge to some positive constants $\rho_{0}$ and $a_{0}$ as $|x| \rightarrow \infty$, respectively, and put

$$
E_{0}=\left(\begin{array}{lll}
\mu_{n} I_{3}, & & 0 \\
& \rho_{0} I_{3}, & \\
0 & & a_{0}
\end{array}\right) .
$$

1.4. Assumptions. We make the following assumptions on $E(x)^{*)}$ :

Assumption (A).

(A.1) $E(x)$ is smooth and positive definite uniformly in $x$;

$$
\begin{gathered}
E(x)-E_{0}=O\left(|x|^{-\delta}\right), \quad \delta>0, \quad \text { as } \quad|x| \rightarrow \infty ; \\
\partial_{x}^{m} E(x)=O\left(|x|^{-(1+\delta)}\right), \quad|m| \geqq 1 .
\end{gathered}
$$

Assumption (B).

$$
\mu_{0} \neq a_{0} \text {. }
$$

*) These assumptions will be weakened in Section 7 . 
Physically, this assumption means that the speed of Alfvèn wave $\left(\left|B_{0}\right|\left(\mu_{0} \rho_{0}\right)^{-1 / 2},\left|B_{0}\right|=1\right)$ and the speed of sound $\left(\left(a_{0} \rho_{0}\right)^{-1 / 2}\right)$ do not equal each other and guarantees that the normal surfaces of the unperturbed system $E_{0}^{-1} L_{0}\left(D_{x}\right)$ have no triple intersecting points (Courant-Hilbert [3], p. 615). Unfortunately, our method developed in [7] cannot be applied to such a case. If $\mu_{0} \neq a_{0}$, then the normal surfaces have at most double intersecting points. From now on, we assume, for brevity, that

$$
\mu_{0}>a_{0} .
$$

1.5. Statement of results. We see that the operator $L\left(x, D_{x}\right)=$ $E(x)^{-1} L_{0}\left(D_{x}\right)$ has a natural self-adjoint realization (denoted by the same symbol $L$ ) in $L_{2,0}^{(7)}$ with the energy scalar product

$$
(\phi, \psi)_{E}=\int_{R^{3}}((E(x) \phi, \psi)) d x,
$$

and that the domain $\mathscr{D}(L)$ is given as

$$
\mathscr{D}(L)=\left\{u ; u \in L_{2,0}^{(7)}, L u \in L_{2,0}^{(7)}\right\} .
$$

Now, we shall state the main results on the spectral properties of the operator $L$. We always assume that Assumptions (A) and (B) are satisfied.

THEOREM 1.1. The non-zero eigenvalues of $L$ are of finite multiplicity and discrete with possible accumulating points 0 and $\pm \infty$.

Theorem 1.2. Assume that $\lambda, \lambda \neq 0$, is not an eigenvalue of $L$. Let $R(\lambda \pm i \kappa)=(L-(\lambda \pm i \kappa))^{-1}, 0<\kappa<1$. Then, the following statements hold:

(i) There exists a constant $C=C_{\alpha}$ independent of $\kappa$ such that for $f \in L_{2, \alpha}^{(i)}, \alpha>1 / 2$,

$$
|R(\lambda \pm i \kappa) f|_{0,-\alpha} \leqq C|f|_{0, \alpha} .
$$

(ii) There exist bounded operators $R(\lambda \pm i 0)$ from $L_{2, \alpha}^{(7)}$ to $L_{2,-\alpha}^{(7)}$ such that $R(\lambda \pm i \kappa)$ converge to $R(\lambda \pm i 0)$ weakly in $L_{2,-\alpha}^{(7)}$ as $\kappa \rightarrow 0$;

$$
\lim _{\kappa \downarrow 0}(R(\lambda \pm i \kappa) f, g)_{E}=(R(\lambda \pm i 0) f, g)_{E}
$$

for any $f$ and $g \in L_{2, \alpha}^{(7)}$. Furthermore, the convergence above is uniform in $\lambda$ when $\lambda$ ranges over a compact interval not containing the eigenvalues of $L$.

THEOREM 1.3. The local energy of solution $u(x, t)$ to the symmetric 
hyperbolic system (1.4) decays as $t \rightarrow \pm \infty$ for all initial data orthogonal to the eigenspace of $L$.

The proof of Theorem 1.2 is based on the following two a priori estimates: Let $u \in L_{2,0}^{(7)}$ be a solution to the equation

$$
L u-(\lambda \pm i \kappa) u=f, \quad 0<\kappa<1, \quad \lambda \neq 0,
$$

with $f \in L_{2, \alpha}^{(7)}, \alpha>1 / 2$. Then, there exists a constant $C$ independent of $\kappa$ such that

$$
\begin{aligned}
& |u|_{0-\alpha} \leqq C\left\{|f|_{0, \alpha}+|u|_{-1,-\alpha}\right\}, \\
& |u|_{0,-\nu} \leqq C\left\{|f|_{0, \alpha}+|u|_{0,-\alpha}\right\}
\end{aligned}
$$

for any $\nu, 1 / 2<\nu<\alpha$. The main part of our argument in the sequel is devoted to the proof of the two a priori estimates above. For notational convenience, the proof is done only for the "+" case and we assume that $\lambda>0$.

1.6. Proof of Theorem 1.3. Though Theorem 1.3 is an immediate consequence of Theorem 1.2, we shall prove it here for the sake of completeness.

Proof of Theorem 1.3. Let $\mathscr{E}(\lambda)$ be the spectral resolution associated with $L ; L=\int \lambda d \mathscr{E}(\lambda)$. Since the total energy of solutions to equation (1.4) is conserved and since $L_{2, \alpha}^{(7)}, \alpha>1 / 2$, is dense in $L_{2,0}^{(7)}$, we have only to prove the decay of local energy for initial data of the form $\mathscr{E}((a, b)) f, f \in L_{2, \alpha}^{(7)}$, where $(a, b)$ is a finite interval not containing the eigenvalues of $L$. First we note that the strong measurability of $R(\lambda \pm i 0) f, f \in L_{2, \alpha}^{(7)}$, in $\lambda$ follows from the weak measurability which is shown by Theorem 1.2, and hence

$$
\mathscr{E}((a, \lambda)) f=(2 \pi i)^{-1} \int_{a}^{\lambda}(R(\lambda+i 0)-R(\lambda-i 0)) f
$$

for $a<\lambda<b$. On the other hand, the solution $u(x, t)$ to the equation (1.4) with initial data $\mathscr{E}((a, b)) f$ is represented as

$$
u(x, t)=\int_{a}^{b} e^{-i t \lambda} d \mathscr{E}(\lambda) f .
$$

Hence, it follows that

$$
u(x, t)=(2 \pi i)^{-1} \int_{a}^{b} e^{-i t \lambda} F(\lambda) d \lambda,
$$


where $F(\lambda)=((R(\lambda+i 0)-R(\lambda-i 0)) f$. Since $F(\lambda)$ can be regarded as an integrable function of $\lambda$ with values in $L_{2,-\alpha}^{(7)}$, the Riemann-Lebesgue theorem completes the proof.

\section{§ 2. Preliminaries}

2.1. Normal surfaces. We define the matrix $L(x, \xi)$ of size $7 \times 7$ as

$$
L(x, \xi)=E(x)^{-1} L_{0}(\xi) .
$$

Then, after a simple but tedious calculation, the seven eigenvalues of $L(x, \xi)$ are given as follows:

(i) zero eigenvalue with simple multiplicity, $\lambda_{0}(x, \xi)=0$;

(ii) not identically zero eigenvalues, $\lambda_{ \pm 1}(x, \xi), \lambda_{ \pm 2}(x, \xi)$;

$$
\begin{aligned}
& \lambda_{ \pm 1}(x, \xi)= \pm\left(\mu_{0} \rho(x)\right)^{-1 / 2} \xi_{1}, \\
& \lambda_{ \pm 2}(x, \xi)= \pm\left(\mu_{0} \rho(x)\right)^{-1 / 2} \sqrt{1 / 2\left((\kappa(x)+1)|\xi|^{2}-|\xi| \sqrt{X(x, \xi)}\right)},
\end{aligned}
$$

where $\kappa(x)=\mu_{0} / a(x)$ and

$$
X(x, \xi)=(\kappa(x)+1)^{2}|\xi|^{2}-4 \kappa(x) \xi_{1}^{2} \quad(\geqq 0) .
$$

(iii) positive eigenvalue, $\lambda_{p}(x, \xi)$;

$$
\lambda_{p}(x, \xi)=\left(\mu_{0} \rho(x)\right)^{-1 / 2} \sqrt{\left.1 / 2(\kappa(x)+1)^{2}|\xi|^{2}+|\xi| \sqrt{X(x, \xi)}\right)} .
$$

(iv) negative eigenvalue, $\lambda_{n}(x, \xi) ; \lambda_{n}(x, \xi)=-\lambda_{p}(x, \xi)$.

Consequently, as is easily seen, the rank of $L(x, \xi)$ is not constant; $\operatorname{rank}(L(x, \xi))=6\left(\right.$ for $\left.\xi_{1} \neq 0\right),=2$ (for $\xi_{1}=0(\xi \neq 0)$ ).

We denote the seven eigenvalues of the unperturbed system $E_{0}^{-1} L_{0}(\xi)$ by $\lambda_{0}^{(0)}(\xi), \lambda_{ \pm j}^{(0)}(\xi)(j=1,2), \lambda_{p}^{(0)}(\xi)$ and $\lambda_{n}^{(0)}(\xi)$. These eigenvalues are expressed by the same relations as above with $\kappa(x)$ and $\rho(x)$ replaced by $\kappa_{0}=\mu_{0} / a_{0}$ and $\rho_{0}$, respectively. We further define the normal surfaces $S_{ \pm j}(j=1,2)$ and $S_{p}$ as follows:

$$
S_{ \pm j}=\left\{\xi ; \lambda_{ \pm j}^{(0)}(\xi)=1\right\}, \quad S_{p}=\left\{\xi ; \lambda_{p}^{(0)}(\xi)=1\right\} .
$$

Then, since $\kappa_{0} \neq 1$ by Assumption (B), we see that all the eigenvalues of $E_{0}^{-1} L_{0}(\xi)$ are smooth functions of $\xi(\xi \neq 0)$, and since $\kappa_{0}>1$ by (1.8), the normal surfaces $S_{+1}$ and $S_{+2}$ (resp. $S_{-1}$ and $S_{-2}$ ) intersect with each other only at $\left(\left(\mu_{0} \rho_{0}\right)^{1 / 2}, 0,0\right)\left(\operatorname{resp} .\left(-\left(\mu_{0} \rho_{0}\right)^{1 / 2}, 0,0\right)\right)$. If $\kappa_{0}=1$, then the three normal surfaces $S_{+1}, S_{+2}$ and $S_{p}\left(S_{-1}, S_{-2}\right.$ and $\left.S_{p}\right)$ intersect with each other at the same point as above (Courant-Hilbert [3] p. 615). 
2.2. Eigenvectors of $L(x, \xi)$. We denote by $e_{0}(x, \xi), e_{ \pm j}(x, \xi)(j=1,2)$, $e_{p}(x, \xi)$ and $e_{n}(x, \xi)$ the normalized eigenvectors of $L(x, \xi)$ corresponding to the eigenvalues $\lambda_{0}(x, \xi), \lambda_{ \pm j}(x, \xi), \lambda_{p}(x, \xi)$ and $\lambda_{n}(x, \xi)$, respectively. Similarly, we denote by $e_{0}^{(0)}(\xi), e_{ \pm j}^{(0)}(\xi)(j=1,2), e_{p}^{(0)}(\xi)$ and $e_{n}^{(0)}(\xi)$ the normalized eigenvectors of the unperturbed system $E_{0}^{-1} L_{0}(\xi)$. Then, it is easily seen that all the eigenvectors are homogeneous of degree zero in $\xi$ and that

$$
e_{0}(x, \xi)=e_{0}^{(0)}(\xi)={ }^{t}\left(\xi_{1}, \xi_{2}, \xi_{3}, 0,0,0,0\right),|\xi|=1 .
$$

LEMMA 2.1. Let $\Omega(\subset\{\xi ;|\xi|=1\})$ be a small neighborhood of $\left\{\xi ; \xi_{1}=0\right.$, $|\xi|=1\}$. Then, $e_{ \pm j}(x, \xi)(j=1,2), e_{p}(x, \xi)$ and $e_{n}(x, \xi)$ can be chosen as smooth functions of $(x, \xi),(x, \xi) \in R_{x}^{3} \times \Omega$, Furthermore, it holds that

$$
\begin{aligned}
& e_{ \pm j}(x, \xi)-e_{ \pm j}^{(0)}(\xi)=O\left(|x|^{-\delta}\right), \\
& \partial_{x}^{m} e_{ \pm j}(x, \xi)=O\left(|x|^{-(1+\delta)}\right), \quad|m| \geqq 1,
\end{aligned}
$$

as $|x| \rightarrow \infty$, uniformly in $\xi, \xi \in \Omega$. Similar estimates also hold for $e_{p}(x, \xi)$ and $e_{n}(x, \xi)$.

Proof. The proof is a direct but tedious calculation, so we give only a sketch. First, it should be noted that the eigenvalues $\lambda_{ \pm j}(x, \xi)(j=1,2)$, $\lambda_{p}(x, \xi)$ and $\lambda_{n}(x, \xi)$ are smooth in $R_{x}^{3} \times \Omega$, if $\Omega$ is chosen small enough. The assertion for $e_{p}(x, \xi)$ and $e_{n}(x, \xi)$ can be easily verified, since the eigenvalues $\lambda_{p}(x, \xi)$ and $\lambda_{n}(x, \xi)$ are simple in $R_{x}^{3} \times \Omega$.

We write the eigenvalue problem $L_{0}(\xi) u=\lambda E(x) u, u={ }^{t}(h, v, p)(h, v$ 3-dimensional vectors, $p$ 1-dimensional vector) as

$$
\begin{aligned}
& H_{0}(\xi) v=\lambda \mu_{0} h, \\
& H_{0}^{*}(\xi) h+A(\xi) p=\lambda \rho(x) v, \\
& A^{*}(\xi) v=\lambda a(x) p,
\end{aligned}
$$

where $H_{0}(\xi)\left(=\Lambda(\xi)\left[B_{0}\right]\right)$ and $A(\xi)$ are defined by $\left(1.6^{\prime}\right)$;

$$
H_{0}(\xi)=\left(\begin{array}{rrr}
0, & \xi_{2}, & \xi_{3} \\
0, & -\xi_{1}, & 0 \\
0, & 0, & -\xi_{1}
\end{array}\right) .
$$

The above problem is readily reduced to

$$
\left(H_{0}^{*}(\xi) H_{0}(\xi)+\kappa(x) A(\xi) A^{*}(\xi)\right) v=\mu v \text {. }
$$

The eigenvalues $\mu_{j}(x, \xi)(j=1,2,3)$ of $(2.6)$ are related to the original eigenvalues $\lambda_{ \pm j}(x, \xi)(j=1,2)$ and $\lambda_{p}(x, \xi)$ by 


$$
\begin{aligned}
& \mu_{j}(x, \xi)=\mu_{0} \rho(x) \lambda_{ \pm j}(x, \xi)^{2}, \quad j=1,2, \\
& \mu_{3}(x, \xi)=\mu_{0} \rho(x) \lambda_{p}(x, \xi)^{2}\left(=\mu_{0} \rho(x) \lambda_{n}(x, \xi)^{2}\right) .
\end{aligned}
$$

Set $e={ }^{t}(1,0,0)\left(=B_{0}\right)$. Then, we see that $\zeta=b(\xi)(\xi \times e)=b(\xi)^{t}\left(0, \xi_{3},-\xi_{2}\right)$, $b(\xi)=\left(\xi_{2}^{2}+\xi_{3}^{3}\right)^{-1 / 2}(\neq 0$ in $\Omega)$, is the eigenvector corresponding to $\mu_{1}(x, \xi)$. Furthermore, $H_{0}(\xi) \zeta=O\left(\left|\xi_{1}\right|\right)$ and $A^{*}(\xi) \zeta=0$. Hence, $e_{ \pm 1}(x, \xi)$ are determined as smooth functions of $(x, \xi)$ by use of relations (2.3) and (2.5).

Let $\eta=b(\xi)^{t}\left(0, \xi_{2}, \xi_{3}\right)$. Obviously, $(e, \zeta, \eta)$ forms an orthonormal system and it holds that $e \times \zeta=\eta, \eta \times e=\zeta, \zeta \times \eta=e$ and $\xi=\xi_{1} e+b(\xi)^{-1} \eta$. We write the eigenvector $w=w(x, \xi)$ corresponding to $\mu_{2}(x, \xi)$ as $w=$ $\alpha(x, \xi) e+\beta(x, \xi) \eta\left(\alpha^{2}+\beta^{2}=1\right)$ and insert it into (2.6). Then, using the relations

$$
\begin{aligned}
& H_{0}^{*}(\xi) H_{0}(\xi) w=e \times \xi \times \xi \times e \times w=\beta|\xi|^{2} \eta, \\
& A(\xi) A^{*}(\xi) w=((\xi, w)) \xi=\left(\alpha \xi_{1}+\beta b(\xi)^{-1}\right)\left(\xi_{1} e+b(\xi)^{-1} \eta\right),
\end{aligned}
$$

we obtain the relation between $\alpha$ and $\beta$;

$$
\kappa(x)\left(\alpha \xi_{1}+\beta b(\xi)^{-1}\right) \xi_{1}=\mu_{2}(x, \xi) \alpha .
$$

Hence, if we note that $\mu_{2}(x, \xi)=O\left(\left|\xi_{1}\right|^{2}\right)$, we can determine $\alpha$ and $\beta$ as smooth functions so that $\alpha=O(1)$ and $\beta=O\left(\left|\xi_{1}\right|\right)$, respectively. Thus, since $\lambda_{ \pm 2}(x, \xi)=O\left(\left|\xi_{1}\right|\right)$, the eigenvector $e_{ \pm 2}(x, \xi)$ can be chosen as smooth functions by use of (2.3) and (2.5).

2.3. Weighted pseudo-differential operators. The next symbol class of pseudo-differential operators has been already introduced in [6].

Definition 2.1. We say that $P(x, \xi)=\left\{p_{j k}(x, \xi)\right\}_{j, k=1, \ell},(x, \xi) \in R_{x}^{3} \times R_{\xi}^{3}$, belongs to $A_{\theta, \sigma}^{(s)}(\ell), \sigma \geqq \theta \geqq 0$, when the following conditions are satisfied:

(a) $p_{j k}(x, \xi)$ is smooth in $R_{x}^{3} \times R_{\xi}^{3}$;

(b) $\left|\partial_{\xi}^{n} p_{j k}(x, \xi)\right| \leqq C_{n}(1+|x|)^{-\theta}(1+|\xi|)^{s-|n|}$;

(c) $\left|\partial_{x}^{m} \partial_{\xi}^{n} p_{j k}(x, \xi)\right| \leqq C_{m, n}(1+|x|)^{-\sigma}(1+|\xi|)^{s-|n|},|m| \geqq 1$.

We say that a family of $P(x, \xi ; \varepsilon)$ with parameter $\varepsilon$ belongs to $A_{\theta, \sigma}^{(s)}(\ell)$ uniformly in $\varepsilon$, if the above constants $C_{n}$ and $C_{m, n}$ are taken uniformly in $\varepsilon$.

We now define the pseudo-differential operator $P\left(x, D_{x}\right)$ with symbol $P(x, \xi) \in A_{\theta, \sigma}^{(s)}(\ell)$ as follows:

$$
P u=(2 \pi)^{-3} \int e^{i x \cdot \xi} P(x, \xi) \hat{u}(\xi) d \xi
$$

for $u(x)={ }^{t}\left(u_{1}(x), \cdots, u_{\ell}(x)\right) \in \mathscr{S}, \mathscr{S}$ being the Schwartz space of rapidly 
decreasing smooth functions, where $\hat{u}(\xi)$ is the Fourier transform of $u$; $\hat{u}(\xi)=\int e^{-i x \cdot \xi} u(x) d x$ and the integration with no domain attached is taken over the whole space.

Definition 2.2. We say that $P\left(x, D_{x}\right) \in O P A_{\theta, \sigma}^{(s)}(\ell)$, when it is a pseudodifferential operator with symbol $P(x, \xi) \in A_{\theta, \sigma}^{(s)}(\ell)$.

For the calculus of pseudo-differential operators of class $O P A_{\theta, \sigma}^{(s)}(\ell)$, the fundamental properties have been formulated as Propositions 2.3 and 2.4 in [6], so we do not state these properties here.

\section{$\S 3$. The proof of a priori estimates, I}

In this section, we consider the equation

$$
L u-(\lambda+i \kappa) u=f, \quad \lambda>0, \quad 0<\kappa<1,
$$

with $f \in L_{2, \alpha}^{(7)}, \alpha>1 / 2$, and prove the a priori estimate (1.10). The proof is rather long and is divided into several steps.

For $\delta$ in Assumption (A.2), we fix $\theta$ so that

$$
0<\theta<\delta
$$

and assume that

$$
\frac{1}{2}<\alpha<\frac{1}{2}(1+\theta)
$$

which obviously loses no generality.

3.1. The first step. Let $\Gamma_{0}(\xi)$ be the projection onto the zero eigenspace (one dimension) of $L_{0}(\xi)$ and let $\Gamma(\xi)=I_{7}-\Gamma_{0}(\xi)$. By (2.2), we see that $\Gamma_{0}(\xi)$ is expressed as

$$
\Gamma_{0}(\xi)=\left(\begin{array}{ccc}
|\xi|^{-2} A(\xi) A^{*}(\xi), & 0, & 0 \\
0, & 0, & 0 \\
0, & 0, & 0
\end{array}\right),
$$

where $A(\xi)$ is defined by $\left(1.6^{\prime}\right)$, and hence both $\Gamma_{0}(\xi)$ and $\Gamma(\xi)$ are commutative with $E(x)$.

Let $Q$ be an open set in $R_{\xi}^{3}$ such that

$$
Q=\left\{\xi=\left(\xi_{1}, \xi_{2}, \xi_{3}\right) ;|\xi|>N,\left|\xi_{1}\right|>d|\xi|\right\},
$$

where $N$ (large enough) and $d(>0$, small enough) are fixed arbitrarily. 
Lemma 3.1. Let $\phi(\xi)\left(\in A_{0,0}^{(0)}(1)\right)$ be a smooth non-negative function with support in $Q$ and let $\tilde{\Gamma}(\xi)=\phi(\xi) \Gamma(\xi)$. Let $u\left(\epsilon L_{2,0}^{(7)}\right)$ be a solution to equation (3.1). Then, $\tilde{\Gamma}\left(D_{x}\right) u$ belongs to $L_{2, \alpha}^{(7)}$ and

$$
\left|\tilde{\Gamma}\left(D_{x}\right) u\right|_{0, \alpha} \leqq C\left\{|f|_{0, \alpha}+|u|_{-1,-\alpha}\right\}
$$

for $C$ independent of $\kappa$.

Proof. We write the equation (3.1) as

$$
L_{0}\left(D_{x}\right) u-\left(\lambda+i_{\kappa}\right) E(x) u=E(x) f .
$$

We set $v=\tilde{\Gamma}\left(D_{x}\right) u$. Then, $v$ obeys the equation

$$
L_{0}\left(D_{x}\right) v-(\lambda+i \kappa) E(x) v=\tilde{\Gamma}\left(D_{x}\right) E(x) f+r
$$

with $r=(\lambda+i \kappa)\left[\tilde{\Gamma}\left(D_{x}\right), E(x)\right] u$, [,] being the commutator. By the commutativity of $\Gamma(\xi)$ with $E(x)$ and by assumption (A.3), we have $\left[\tilde{\Gamma}\left(D_{x}\right), E(x)\right]$ $\in O P A_{\sigma, \sigma}^{(-1)}(7), \sigma=1+\theta$, and hence it follows from assumption (3.3) that

$$
|r|_{0, \nu+\alpha} \leqq C_{\nu}|u|_{-1, \nu-\alpha}, \quad 0 \leqq \nu \leqq \alpha .
$$

If we take $N$ large enough in (3.4), then $L_{0}(\xi)-\left(\lambda+i_{\kappa}\right) E(x)$ is invertible in $\mathcal{O}=R_{x}^{3} \times Q$ uniformly in $\kappa$ and in $(x, \xi)(\in \mathcal{O})$, and we have

$$
\left|L_{0}(\xi) p\right|^{2} \geqq C|\xi|^{2}|\Gamma(\xi) p|^{2}, \quad \xi \in Q,
$$

for any $p \in C^{7}$. This proves the lemma.

Lemma 3.2. Let $\phi(\xi)$ be as in Lemma 3.1 and let $\tilde{\Gamma}_{0}(\xi)=\phi(\xi) \Gamma_{0}(\xi)$. Then,

$$
\left|\tilde{\Gamma}_{0}\left(D_{x}\right) u\right|_{0, \alpha} \leqq C|f|_{0, \alpha} .
$$

Proof. We start with equation (3.5). Set $v=\tilde{\Gamma}_{0}\left(D_{x}\right) u$. Then, since $\tilde{\Gamma}_{0}\left(D_{x}\right)$ is commutative with $E(x)$, we have

$$
-(\lambda+i \kappa) E(x) v=\tilde{\Gamma}_{0}\left(D_{x}\right) E(x) f .
$$

This proves the lemma.

We now combine Lemma 3.2 with Lemma 3.1 to obtain the following result.

Lemma 3.3. Let $\phi(\xi)$ be as in Lemma 3.1. Then,

$$
\left|\phi\left(D_{x}\right) u\right|_{0, \alpha} \leqq C\left\{|f|_{0, \alpha}+|u|_{-1,-\alpha}\right\}
$$

for $C$ independent of $\kappa$. 
3.2. The second step. Let $G$ be an open set in $R_{\xi}^{3}$ such that

$$
G=\left\{\xi ;|\xi|>N,\left|\xi_{1}\right|<d|\xi|\right\},
$$

where $N$ and $d$ are fixed arbitrarily as before. Let $\psi(\xi)\left(\in A_{0,0}^{(0)}(1)\right)$ be a smooth non-negative function with support in $G$. Then, $\psi\left(D_{x}\right) u$ satisfies the equation

$$
L_{0}\left(D_{x}\right) \psi\left(D_{x}\right) u-(\lambda+i \kappa) E(x) \psi\left(D_{x}\right) u=\psi\left(D_{x}\right) E(x) f+r,
$$

where $r=\left(\lambda+i_{\kappa}\right)\left[\psi\left(D_{x}\right), E(x)\right] u$ satisfies an estimate similar to (3.7) with another constant $C$.

Here we introduce new notations. 1) If $\chi(\xi) \psi(\xi)=\psi(\xi)$ for a smooth non-negative function $\chi(\xi)\left(\in A_{0,0}^{(0)}(1)\right)$ with support in $G$, then we write $\chi \gg \psi$. 2) We denote by $r=r(x), r={ }^{t}\left(r_{1}, \cdots, r_{7}\right)$, all terms satisfying estimates of the same type as in (3.7) with another constant $C$.

We now write the differential operator $E(x)^{-1 / 2} L_{0}\left(D_{x}\right) E(x)^{-1 / 2}$ as

$$
E(x)^{-1 / 2} L_{0}\left(D_{x}\right) E(x)^{-1 / 2}=\tilde{L}\left(x, D_{x}\right)+B(x),
$$

where the symbol $\tilde{L}(x, \xi)$ of $\tilde{L}\left(x, D_{x}\right)$ is $E(x)^{-1 / 2} L_{0}(\xi) E(x)^{-1 / 2}$ and $B(x)$ satisfies $\partial_{x}^{m} B(x)=O\left(|x|^{-(1+\delta)}\right),|m| \geqq 0$, by assumption (A.3). We set

$$
\tilde{u}=E(x)^{1 / 2} \psi\left(D_{x}\right) u \text {. }
$$

Then, $\tilde{u}$ obeys the equation

$$
\tilde{L}\left(x, D_{x}\right) \tilde{u}+B(x) \tilde{u}-(\lambda+i \kappa) \tilde{u}=E(x)^{-1 / 2} \psi\left(D_{x}\right) E(x) f+r .
$$

We want to diagonalize the equation (3.10). By Lemma 2.1, there exists a unitary matrix $U(x, \xi)$ smooth in $\mathscr{N}=R_{x}^{3} \times G$ such that in $\mathscr{N}$

$$
U(x, \xi) \tilde{L}(x, \xi) U^{*}(x, \xi)=\mathscr{D}(x, \xi),
$$

where $\mathscr{D}(x, \xi)$ is a diagonal matrix of the following form:

$$
\mathscr{D}(x, \xi)=\left(\begin{array}{cccccc}
\lambda_{0}, & & & & \\
& \lambda_{p}, & & & 0 & \\
& \lambda_{n}, & & & \\
& & \lambda_{+1}, & & & \\
& & & \lambda_{+2}, & & \\
& & & & \lambda_{-1}, & \\
& & & & \lambda_{-2}
\end{array}\right) .
$$

Let $\chi \gg \psi$ and we define $\tilde{U}(x, \xi)$ as $\tilde{U}(x, \xi)=\chi(\xi) U(x, \xi)$, which belongs 
to $A_{0, \sigma}^{(0)}(7), \sigma=1+\theta$. (From now on, we use $\sigma$ with the meaning ascribed here.) We define $\tilde{U}^{*}(x, \xi)$ similarly. For $\tilde{u}$ defined by (3.9), we set

$$
v=\tilde{U}\left(x, D_{x}\right) \tilde{u} \text {. }
$$

Lemma 3.4. Let $v$ be as above. Then,

$$
\tilde{u}=\tilde{U}_{*}\left(x, D_{x}\right) v+R\left(x, D_{x}\right) v+\tilde{r},
$$

where $\tilde{U}_{*}\left(x, D_{x}\right)$ is the pseudo-differential operator with symbol $\tilde{U}^{*}(x, \xi)$, while $R\left(x, D_{x}\right) \in O P A_{\sigma, \sigma}^{(-1)}(7)$ and $\tilde{r}$ satisfies the estimate $|\tilde{r}|_{1, \nu+a} \leqq C_{\nu}|u|_{-1, \nu-\alpha}$, $0 \leqq \nu \leqq \alpha$.

Proof. We may write

$$
\tilde{U}_{*}\left(x, D_{x}\right) v=\left(\chi_{2}\left(D_{x}\right)+R_{0}\left(x, D_{x}\right)\right) E(x)^{1 / 2} \psi\left(D_{x}\right) u
$$

with $\left.R_{0}\left(x, D_{x}\right) \in O P A_{\sigma, \sigma}^{(-1)}\right)(7)$, where $\chi_{2}(\xi)=\chi(\xi)^{2}$. Since $\chi \gg \psi(\chi(\xi)=1$ on the support of $\psi),\left[\chi_{2}\left(D_{x}\right), E(x)^{1 / 2}\right] \psi\left(D_{x}\right)$ belongs to $O P A_{\sigma, \sigma}^{(-s)}(7)$ for any $s$ (large enough), and hence we have

$$
\tilde{u}=\tilde{U}_{*}\left(x, D_{x}\right) v-R_{0}\left(x, D_{x}\right) \tilde{u}+\tilde{r} .
$$

We have only to insert this expression into $\tilde{u}$ in the second term on the right side. Thus, the proof is completed.

LEMma 3.5. We have

$$
\chi\left(D_{x}\right) v=v+e,
$$

where $e$ satisfies the estimate $|e|_{s, \nu+\alpha} \leqq C_{s, \nu}|u|_{-1, \nu-\alpha}, 0 \leqq \nu \leqq \alpha$, for any $s$ large enough.

Proof. The proof is easily done by use of the relation $\chi \gg \psi$.

Now, we diagonalize the equation (3.10). We let $\tilde{U}\left(x, D_{x}\right)$ operate on equation (3.10) and insert the expression for $\tilde{u}$ in Lemma 3.4 into the resulting equation. Then, we obtain, using Lemma 3.5, an equation of the diagonalized form;

$$
\left(\mathscr{D}\left(x, D_{x}\right)-(\lambda+i \kappa)\right) \chi\left(D_{x}\right) v+T\left(x, D_{x}\right) v=g+r,
$$

where $T\left(x, D_{x}\right) \in O P A_{\sigma, \sigma}^{(0)}(7)$, and $g$ is expressed as

$$
g=\tilde{U}\left(x, D_{x}\right) E(x)^{-1 / 2} \psi\left(D_{x}\right) E(x) f .
$$

It is convenient in the discussion below to write a vector-valued function $h=h(x)$ with seven components as 


$$
h={ }^{t}\left(h_{0}, h_{p}, h_{n}, \hat{h}\right), \quad \hat{h}={ }^{t}\left(h_{+1}, h_{+2}, h_{-1}, h_{-2}\right) .
$$

LEMMA 3.6. $v_{p}$ satisfies the estimate

$$
\left|v_{p}\right|_{0, \nu}^{(1)} \leqq C_{\nu}\left\{|f|_{0, \nu}+|u|_{-1,-\alpha}\right\}, \quad-\alpha \leqq \nu \leqq \alpha ;
$$

similarly for $v_{n}$.

Proof. By (3.13), $v_{p}$ obeys the equation

$$
\left(\lambda_{p}\left(x, D_{x}\right)-\left(\lambda+i_{\kappa}\right)\right) \chi\left(D_{x}\right) v_{p}=g_{p}-h_{p}+r_{p},
$$

where $h=T\left(x, D_{x}\right) v$. Since $\lambda_{p}(x, \xi)-(\lambda+i \kappa)$ is invertible in $\mathscr{N}=R_{x}^{3} \times G$ uniformly in $\kappa$, we obtain the desired estimate immediately. The proof for $v_{n}$ is also done in a similar way.

LEMMA 3.7. $v_{0}$ satisfies the estimate

$$
\left|v_{0}\right|_{0, \nu}^{(1)} \leqq C_{\nu}\left\{|f|_{0, \nu}+|u|_{-1,-\alpha}\right\}, \quad-\alpha \leqq \nu \leqq \alpha .
$$

Proof. We write $\tilde{u}=E(x)^{1 / 2} \psi\left(D_{x}\right) u$ as $\tilde{u}={ }^{t}\left(\tilde{u}_{1}, \cdots, \tilde{u}_{7}\right)$. As is easily seen from (2.2), $e_{0}(x, \xi)=e_{0}^{(0)}(\xi)=|\xi|^{-1} t\left(\xi_{1}, \xi_{2}, \xi_{3}, 0,0,0,0\right)$ is the normalized eigenvector of $\tilde{L}(x, \xi)$ corresponding to the identically zero eigenvalue. Thus, we have

$$
v_{0}=\sum_{j=1}^{3} a_{j}\left(D_{x}\right) u_{j}
$$

where $a_{j}(\xi)=\chi(\xi) \xi_{j} /|\xi|$. Hence, it follows from the definition of $\Gamma_{0}\left(D_{x}\right)$ that

$$
\chi_{2}\left(D_{x}\right) \Gamma_{0}\left(D_{x}\right) \tilde{u}={ }^{t}\left(a_{1}\left(D_{x}\right) v_{0}, a_{2}\left(D_{x}\right) v_{0}, a_{3}\left(D_{x}\right) v_{0}, 0,0,0,0\right) .
$$

This implies that

$$
\chi_{2}\left(D_{x}\right) v_{0}=\sum_{j=1}^{3} a_{j}\left(D_{x}\right)\left[\chi_{2}\left(D_{x}\right) \Gamma_{0}\left(D_{x}\right) \tilde{u}\right]_{j},
$$

where $\left[\chi_{2}\left(D_{x}\right) \Gamma_{0}\left(D_{x}\right) \tilde{u}\right]_{j}$ denotes the $j$-th component. We can write (3.15) as

$$
\chi_{2}\left(D_{x}\right) v_{0}=\sum_{j=1}^{3} a_{j}\left(D_{x}\right)\left[E(x)^{1 / 2} \tilde{\Gamma}_{0}\left(D_{x}\right) u\right]_{j}
$$

because of the commutativity of $\chi_{2}\left(D_{x}\right) \Gamma_{0}\left(D_{x}\right)$ with $E(x)^{1 / 2}$, where $\tilde{\Gamma}_{0}(\xi)=$ $\psi(\xi) \Gamma_{0}(\xi)$. By the same argument as in the proof of Lemma 3.2, it can be verified that $\left|\tilde{\Gamma}_{0}\left(D_{x}\right) u\right|_{0, \nu} \leqq C_{\nu}|f|_{0, \nu},(-\alpha \leqq \nu \leqq \alpha)$, and hence we obtain the desired estimate by use of Lemma 3.5.

We combine Lemmas 3.6 and 3.7 to obtain the equation for $\hat{v}$; 


$$
\left(\hat{\mathscr{D}}\left(x, D_{x}\right)-(\lambda+i \kappa)\right) \chi\left(D_{x}\right) \hat{v}+\hat{T}\left(x, D_{x}\right) \hat{v}=\hat{g}+q,
$$

where $\hat{T}\left(x, D_{x}\right) \in O P A_{\sigma, \sigma}^{(0)}(4)$ and $\hat{\mathscr{D}}(x, \xi)$ is of the form

$$
\hat{\mathscr{D}}(x, \xi)=\left(\begin{array}{cccc}
\lambda_{+1}, & & 0 \\
& \lambda_{+2}, & & \\
0 & \lambda_{-1}, & \\
0 & & \lambda_{-2}
\end{array}\right)
$$

while $q$ satisfies the estimate

$$
|q|_{0, \nu+\alpha}^{(4)} \leqq C_{\nu}\left\{|f|_{0, \nu-\alpha}+|u|_{-1, \nu-\alpha}\right\}, \quad 0 \leqq \nu \leqq \alpha .
$$

3.3. The third step. We further continue the reduction for equation (3.16). Since it is convenient in the discussion below to clarify the separation of the variable $x_{1}$ from $\left(x_{2}, x_{3}\right)$, we write $t=x_{1}$ and $y=\left(x_{2}, x_{3}\right)$, and denote by $\xi=(\tau, \eta), \eta=\left(\eta_{2}, \eta_{3}\right)$, the coordinates dual to $x=(t, y)$. Let $\Pi$ be an open set in $R_{\eta}^{2}$ such that

$$
\Pi=\{\eta ;|\eta|>N / 2\},
$$

$N$ being as in (3.8). We can take $d$ in (3.8) so small that $G \subset\{(\tau, \eta)$; $\eta \in \Pi\}$.

Lemma 3.8. There exist functions $\sigma_{ \pm j}(x, \eta), 1 \leqq j \leqq 2$, smooth in $\mathscr{M}=$ $R_{x}^{3} \times \Pi$ such that in $\mathscr{N}=R_{x}^{3} \times G$

$$
\lambda_{ \pm j}(x, \xi)-\lambda=k_{ \pm j}(x, \xi)\left(\tau-\sigma_{ \pm j}(x, \eta)\right)
$$

with $k_{ \pm j}(x, \xi)\left(k_{+j}>0, k_{-j}<0\right)$ smooth in $\mathscr{N}$. Furthermore, $\sigma_{ \pm j}(x, \eta)$ satisfy the estimate

$$
\begin{aligned}
& \left|\partial_{\eta}^{n} \sigma_{ \pm j}(x, \eta)\right| \leqq C_{n}(1+|\eta|)^{-|n|}, \\
& \left|\partial_{x}^{m} \partial_{\eta}^{n} \sigma_{ \pm j}(x, \eta)\right| \leqq C_{m n}(1+|x|)^{-(1+o)}(1+|\eta|)^{-|n|},|m| \geqq 1,
\end{aligned}
$$

and $\tilde{\chi}(\xi) k_{ \pm j}(x, \xi)$ belongs to $A_{0, \sigma}^{(0)}(1)$ for any $\tilde{\chi}(\xi)\left(\in A_{0,0}^{(0)}(1)\right)$ with support in $G$.

Proof. For $j=1$, it follows from the definition of $\lambda_{ \pm 1}(x, \xi)$ that $\lambda_{ \pm 1}(x, \xi)$ $-\lambda= \pm\left(\mu_{0} \rho(x)\right)^{-1 / / 2}\left(\tau \mp\left(\mu_{0} \rho(x)\right)^{1 / 2} \lambda\right)$. For $j=2$, we solve the equation $\lambda_{ \pm 2}(x, \xi)$ $=\lambda$ for $\tau$. After a direct calculation, the roots $\sigma_{ \pm 2}(x, \eta)$ are obtained as

$$
\sigma_{ \pm 2}(x, \eta)= \pm \sqrt{2 b\left(a+\left(a^{2}+4 \kappa b\right)^{1 / 2}\right)^{-1}}
$$

where 


$$
\begin{aligned}
& a=a(x, \eta)=\kappa(x)|\eta|^{2}-\mu_{0} \rho(x)(\kappa(x)+1) \lambda^{2}, \\
& b=b(x, \eta)=\left(\mu_{0} \rho(x)(\kappa(x)+1)|\eta|^{2}-\mu_{0}^{2} \rho(x)^{2} \lambda^{2}\right) \lambda^{2} .
\end{aligned}
$$

We define $k_{ \pm 2}(x, \xi)$ as

$$
k_{ \pm 2}(x, \xi)=\int_{0}^{1}\left(\partial_{z} \lambda_{ \pm 2}\right)\left(x, \theta \tau+(1-\theta) \sigma_{ \pm 2}(x, \eta), \eta\right) d \theta .
$$

Then, $\lambda_{ \pm 2}(x, \xi)-\lambda=k_{ \pm 2}(x, \xi)\left(\tau-\sigma_{ \pm 2}(x, \eta)\right)$. It is easily verified that $\sigma_{ \pm 2}(x, \eta)$ satisfy the required estimates. Furthermore, since $\partial_{z} \lambda_{+2}(x, \xi)>0$ for $d$ (in (3.8)) small enough and since $C_{1}(1+|\eta|) \leqq(1+|\xi|) \leqq C_{2}(1+|\eta|)$ in $G$, we see that $k_{ \pm 2}(x, \xi)>0$ in $\mathscr{N}$ and that $\tilde{\chi}(\xi) k_{+2}(x, \xi) \in A_{0, \sigma}^{(0)}(1)$ for any $\tilde{\chi}(\xi)$ $\left(\in A_{0,0}^{(0)}(1)\right.$ ) with support in $G$. The proof for $k_{-2}(x, \xi)$ is also done in a similar way. Thus, the proof is completed.

Now, with $k_{ \pm \jmath}(x, \xi)$ in the above lemma, we define the symbols $H(x, \xi)$ and $K(x, \xi)\left(\in A_{0, \sigma}^{(0)}(4)\right)$ as follows:

$$
\begin{aligned}
& H(x, \xi)=\chi(\xi)\left(\begin{array}{cccc}
k_{+1}^{1 / 2}, & & & 0 \\
& k_{+2}^{1 / 2}, & & \\
& 0 & \left(-k_{-1}\right)^{1 / 2}, & \\
& & & \left(-k_{-2}\right)^{1 / 2}
\end{array}\right),
\end{aligned}
$$

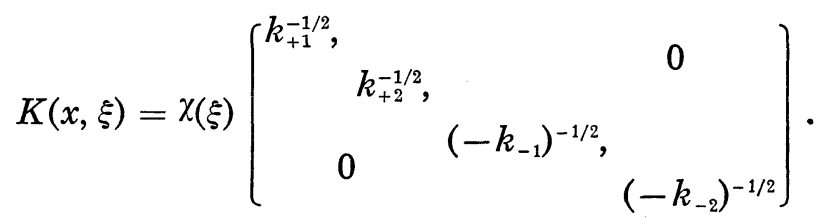

We further define $w$ as

$$
w=H\left(x, D_{x}\right) \hat{v}
$$

LEMma 3.9. We have

$$
\hat{v}=K\left(x, D_{x}\right) w+R_{1}\left(x, D_{x}\right) w+\tilde{r}
$$

with $R_{1}\left(x, D_{x}\right) \in O P A_{\sigma, \sigma}^{(-1)}(4)$, where $\tilde{r}$ satisfies the estimate $|\tilde{r}|_{1, \nu+\alpha}^{(4)} \leqq C|u|_{-1, \nu-\alpha}$, $0 \leqq \nu \leqq \alpha$.

Proof. The proof is done by the same argument as in the proof of Lemmas 3.4 and 3.5.

Let $\omega(\eta)$ be a smooth non-negative function with support in $\Pi$ such that $\omega(\eta)=1$ for $|\eta| \rightarrow \infty$ and $\chi(\xi) \omega(\eta)=\chi(\xi)$. Define the symbol $A(x, \eta)$ as 


$$
A(x, \eta)=\omega(\eta)\left[\begin{array}{ccc}
\sigma_{+1}, & & 0 \\
& \sigma_{+2}, & \\
0 & \sigma_{-1}, & \\
0 & & \sigma_{-2}
\end{array}\right]
$$

for $\sigma_{ \pm j}(x, \eta)$ in Lemma 3.8. Then, by Lemma 3.9, we see from (3.16) that $w$ satisfies the equation

$$
\left(D_{t}-A\left(x, D_{y}\right)\right) w+S\left(x, D_{x}\right) w-i \kappa J K\left(x, D_{x}\right)^{2} w=h+q
$$

with another $q$ satisfying an estimate similar to (3.17), where $S\left(x, D_{x}\right) \in$ $O P A_{\sigma, \sigma}^{(0)}(4), h$ is given as $h=J K\left(x, D_{x}\right) \hat{g}$ and

$$
J=\left(\begin{array}{cc}
I_{2}, & 0 \\
0 & -I_{2}
\end{array}\right) \text {. }
$$

Summing up, we obtain the following result.

LEMMA 3.10. Let $u$ be a solution to equation (3.1) with $f \in L_{2, \alpha}^{(7)}, 1 / 2<$ $\alpha<(1 / 2)(1+\theta)$. Set

$$
w=H\left(x, D_{x}\right)\left[\tilde{U}\left(x, D_{x}\right) E(x)^{1 / 2} \psi\left(D_{x}\right) u\right]^{\wedge},
$$

where [ ] denotes the last four components of $\tilde{U}\left(x, D_{x}\right) E(x)^{1 / 2} \psi\left(D_{x}\right) u$ (7dimensional vector). Then, $w$ satisfies the equation (3.21) with $h$, where $h$ is given by

$$
h=J K\left(x, D_{x}\right)\left[\tilde{U}\left(x, D_{x}\right) E(x)^{-1 / 2} \psi\left(D_{x}\right) E(x) f\right]^{\wedge} .
$$

Equation (3.21) is our basic equation from which the a priori estimate (1.10) is derived.

3.4. The fourth step. Let $\mathscr{H}=L_{2,0}^{(4)}\left(R_{y}^{2}\right)$. Denote by $\langle$,$\rangle and \|\|$ the scalar product and norm in $\mathscr{H}$, respectively. We regard $w=w(t, \cdot)$ defined by (3.22) as a function with values in $\mathscr{H}$ and take the scalar product $\langle$,$\rangle of -i k(t) w$ with equation (3.21), where

$$
k(t)=\exp \left(-2 M \int_{-\infty}^{t}\left(1+s^{2}\right)^{-\alpha} d s\right)
$$

and $M, M>0$, is a constant (large enough) to be determined later. We further take the real part of both sides of the resulting relation and obtain

$$
\frac{1}{2}\left\{\partial_{t}\langle w, k(t) w\rangle-\left\langle\left(\partial_{t} k(t)\right) w, w\right\rangle\right\}+\operatorname{Im}\left\langle A\left(x, D_{y}\right) w, k(t) w\right\rangle=\sum_{j=1}^{3} J_{j}(t),
$$


where

$$
\begin{aligned}
& J_{1}(t)=-\operatorname{Im}\{\langle h, k(t) w\rangle+\langle q, k(t) w\rangle\}, \\
& J_{2}(t)=-\kappa \operatorname{Re}\left\langle J K\left(x, D_{x}\right)^{2} w, k(t) w\right\rangle, \\
& J_{3}(t)=\operatorname{Im}\left\langle S\left(x, D_{x}\right) w, k(t) w\right\rangle .
\end{aligned}
$$

We integrate $(3.24)$ over $(-\infty, \infty)$ with respect to $t$ to obtain

$$
\int_{-\infty}^{\infty}\left\{M\left(1+t^{2}\right)^{-\alpha}\left\|k_{1}(t) w\right\|^{2}+\operatorname{Im}\left\langle A\left(x, D_{y}\right) w, k(t) w\right\rangle\right\} d t=\sum_{j=1}^{3} T_{j},
$$

where $k_{1}(t)=k(t)^{1 / 2}$ and $T_{j}=\int_{-\infty}^{\infty} J_{j}(t) d t$.

We shall estimate each term $T_{j}$ on the right side of (3.25). Since $|h|_{0, \alpha}^{(4)} \leqq C|f|_{0, \alpha}$ by the definition (3.23) of $h$ and since $|q|_{0, \alpha}^{(4)} \leqq C\left\{|f|_{0, \alpha}+|u|_{-1,-\alpha}\right\}$, we have

$$
\left|T_{1}\right| \leqq C\left\{|f|_{0, \alpha}+|u|_{-1,-\alpha}\right\}|u|_{0,-\alpha}
$$

for $C$ independent of $M$, because $k(t)$ is bounded uniformly in $M$. $K\left(x, D_{x}\right)$ is of class $O P A_{0, \sigma}^{(0)}(4)$ and hence it is a bounded operator from $L_{2,0}^{(4)}\left(R_{x}^{3}\right)$ to itself. Hence, the term $T_{2}$ is estimated as $\left|T_{2}\right| \leqq \kappa C\left(|u|_{0,0}\right)^{2}$. On the other hand, it follows from equation (3.1) that $\kappa\left(|u|_{0,0}\right)^{2} \leqq C|f|_{0, \alpha}|u|_{0,-\alpha}$. Thus, we have

$$
\left|T_{2}\right| \leqq C|f|_{0, \alpha}|u|_{0,-\alpha} .
$$

The term $T_{3}$ is estimated as follows. $S\left(x, D_{x}\right)$ is of class $O P A_{\sigma, \sigma}^{(0)}(4)$ and hence $\left[k_{1}(t), S\left(x, D_{x}\right)\right]$ is of class $O P A_{\sigma, \sigma}^{(-1)}(4)$. Hence, we have

$$
\left|T_{3}\right| \leqq C_{1} \int_{-\infty}^{\infty}\left(1+t^{2}\right)^{-\alpha}\left\|k_{1}(t) w\right\|^{2} d t+K_{M}|u|_{-1,-\alpha}|u|_{0,-\alpha}
$$

for $C_{1}$ independent of $M . \quad\left(K_{M}\right.$ may depend on $M$.)

Next, we shall estimate the term $T_{4}=\operatorname{Im} \int_{-\infty}^{\infty}\left\langle A\left(x, D_{y}\right) w, k(t) w\right\rangle d t$ on the left side of (3.25). $A(x, \eta)$ is a diagonal matrix with real elements and hence it is symmetric. Therefore, by estimate (3.19) in Lemma 3.8, we see that

$$
\left|T_{4}\right| \leqq C_{2} \int_{-\infty}^{\infty}\left(1+t^{2}\right)^{-\alpha}\left\|k_{1}(t) w\right\|^{2} d t
$$

for $C_{2}$ independent of $M$.

Since the constants $C_{1}$ in (3.26) and $C_{2}$ in (3.27) are independent of 
$M$, we can take $M$ so large that $M-\left(C_{1}+C_{2}\right)>1$. Hence, if $M$ is fixed as above, then we have

$$
\int_{-\infty}^{\infty}\left(1+t^{2}\right)^{-\alpha}\|w(t)\|^{2} d t \leqq C\left\{|f|_{0, \alpha}+|u|_{-1,-\alpha}\right\}|u|_{0, \alpha} .
$$

Now, we shall prove the a priori estimate (1.10).

THEOREM 3.1. Let $u\left(\in L_{2,0}^{(7)}\right)$ be a solution to equation (3.1) with $f \in L_{2, \alpha}^{(7)}$, $1 / 2<\alpha<(1 / 2)(1+\theta)$. Then, there exists a constant $C$ independent of $\kappa$, $0<\kappa<1$, for which (1.10) holds.

Remarks. (i) It is easily seen that the constant $C$ above is also independent of $\lambda$, when $\lambda$ ranges over a compact interval not containing the origin. (ii) Estimate (1.10) is still valid for $\kappa=0$, if it is assumed that a solution to (3.1) with $\kappa=0$ belongs to $L_{2,0}^{(7)}$.

Proof. First, by (3.28)

$$
\left(|w|_{0, \alpha}^{(4)}\right)^{2} \leqq C\left\{|f|_{0, \alpha}+|u|_{-1,-\alpha}\right\}|u|_{0,-\alpha} .
$$

Hence, it follows from Lemmas 3.6, 3.7 and 3.9 that

$$
\left(|v|_{0,-\alpha}\right)^{2} \leqq C\left\{|f|_{0 \alpha}+|u|_{-1,-\alpha}\right\}\left\{|f|_{0, \alpha}+|u|_{0,-\alpha}\right\} .
$$

Furthermore, by Lemma 3.4, we see that $\left(\left|\psi\left(D_{x}\right) u\right|_{0,-\alpha}\right)^{2}$ is majorized by the same bound as above with another constant $C$. Thus, we combine this fact with Lemma 3.3 to obtain the desired estimate and the proof is now completed.

3.5. A priori estimate for the eigenfunctions. The next theorem is used in the proof of Theorem 1.1.

TheOREM 3.2. Let $u\left(\in L_{2,0}^{(7)}\right)$ be an eigenfunction of the operator $L$ with eigenvalue $\lambda, \lambda \neq 0 ; L u=\lambda u$. Then, there exists a constant $C$ such that $|u|_{0,0} \leqq C|u|_{-1,0}$, where the constant $C$ is independent of $\lambda \in K, K$ being $a$ compact interval not containing the origin.

Proof. We start with equation (3.21) with $\kappa=0$ and $h=0$;

$$
\left(D_{t}-A\left(x, D_{y}\right)\right) w+S\left(x, D_{x}\right) w=q,
$$

where $q$ satisfies the estimate

$$
|q|_{0, \nu+\alpha}^{(4)} \leqq C_{\nu}|u|_{-1, \nu-\alpha}, \quad 0 \leqq \nu \leqq \alpha .
$$

We take the scalar product $\langle$,$\rangle in \mathscr{H}$ between equation $\left(3.21^{\prime}\right)$ and $i w$, 
and the real part of both sides of the resulting relation;

$$
-\frac{1}{2} \partial_{t}\langle w, w\rangle=\sum_{j=1}^{3} X_{j}(t)
$$

where

$$
\begin{aligned}
& X_{1}(t)=\operatorname{Im}\left\langle A\left(x, D_{y}\right) w, w\right\rangle, \quad X_{2}(t)=\operatorname{Im}\langle q, w\rangle \quad \text { and } \\
& X_{3}(t)=-\operatorname{Im}\left\langle S\left(x, D_{x}\right) w, w\right\rangle .
\end{aligned}
$$

Integrating $(3.30)$ over $(s, \infty)$ with respect to $t$ gives

$$
\frac{1}{2}\|w(s)\|^{2}=\sum_{j=1}^{3} Y_{j}(s)
$$

where $Y_{j}(s)=\int_{s}^{\infty} X_{j}(t) d t$. We further integrate (3.31) over $(T, \infty)$ with respect to $s$ and obtain

$$
\frac{1}{2} \int_{T}^{\infty}\|w(s)\|^{2} d s=\sum_{j=1}^{3} Z_{j},
$$

where $Z_{j}=\int_{T}^{\infty} Y_{j}(s) d s$.

We make an estimate of each term $Z_{j}$. First, $Z_{1}$ is estimated as

$$
\left|Z_{1}\right| \leqq C T^{1-2 \alpha} \int_{T}^{\infty}\|w(t)\|^{2} d t
$$

since $\left|X_{j}(t)\right| \leqq C\left(1+t^{2}\right)^{-\alpha}\|w(t)\|^{2}$. Next, we have

$$
Z_{2}=\int_{T}^{\infty}(t-T) \operatorname{Im}\langle q, w\rangle d t .
$$

Hence, by the Schwarz inequality and by (3.29), it follows that

$$
\left|Z_{2}\right| \leqq C\left\{T^{1-2 \alpha} \int_{T}^{\infty}\|w(t)\|^{2} d t+\left(|u|_{-1,0}\right)^{2}\right\}
$$

Similarly, for any $\varepsilon(>0)$ small enough, we have

$$
\left|Z_{3}\right| \leqq C_{\varepsilon} T^{1-2 \alpha} \int_{T}^{\infty}\|w(t)\|^{2} d t+\varepsilon\left(|u|_{0,0}\right)^{2},
$$

since $S\left(x, D_{x}\right)$ belongs to $O P A_{\sigma, o}^{(0)}(4)$. Thus, for any $\varepsilon(>0)$ small enough, there exists a constant $T=T(\varepsilon)$ such that

$$
\int_{T}^{\infty}\|w(t)\|^{2} d t \leqq \varepsilon\left(|u|_{0,0}\right)^{2}+C\left(|u|_{-1,0}\right)^{2}
$$


By the same argument as above, we have

$$
\int_{-\infty}^{-T}\|w(t)\|^{2} d t \leqq \varepsilon\left(|u|_{0,0}\right)^{2}+C\left(|u|_{-1,0}\right)^{2}
$$

On the other hand, we combine (3.28) with (1.10) to obtain

$$
\int_{-T}^{T}\|w(t)\|^{2} d t \leqq C_{\varepsilon}\left(|u|_{-1,0}\right)^{2}
$$

for $T=T(\varepsilon)$ fixed above. Hence, by the same reasoning as in the proof of Theorem 3.1, it follows from (3.32) and (3.33) that

$$
\left(|u|_{0,0}\right)^{2} \leqq \varepsilon C\left(|u|_{0,0}\right)^{2}+C_{\varepsilon}\left(|u|_{-1,0}\right)^{2}
$$

for any $\varepsilon(>0)$ small enough. This completes the proof.

\section{$\S 4$. The proof of a priori estimates, II}

In this section, we continue to consider the equation (3.1) and prove the $a$ priori estimate (1.11) by applying the results obtained in [6] on the basis of Agmon's idea.

4.1. Decomposition. Let $\theta$ be as in (3.2). Then, we can decompose $E(x)$ as follows: For any $\varepsilon(>0)$ small enough, there exists a constant $R=R(\varepsilon, \theta)$ such that: (i) $E(x)=E(x ; \varepsilon)+E_{r}(x ; \varepsilon)$; (ii) $E(x)=E(x ; \varepsilon)$ for $|x|>R$, and hence $E_{r}(x ; \varepsilon)$ is of compact support; (iii) $\left|E(x ; \varepsilon)-E_{0}\right|$ $\leqq \varepsilon C(1+|x|)^{-\theta}$ and $\left|\partial_{x}^{m} E(x ; \varepsilon)\right| \leqq \varepsilon C_{m}(1+|x|)^{-\sigma},|m| \geqq 1(\sigma=1+\theta)$, for all $x$.

We rewrite the equation (3.1) as

$$
L_{0}\left(D_{x}\right) u-(\lambda+i \kappa) E(x ; \varepsilon) u=E(x) f+(\lambda+i \kappa) E_{r}(x ; \varepsilon) u
$$

and, for notational convenience, we denote by $f$ the terms on the right side again; $f=E(x) f+(\lambda+i \kappa) E_{r}(x ; \varepsilon) u$.

4.2. Reduction. We start with the equation

$$
L_{0}\left(D_{x}\right) u-\left(\lambda+i_{\kappa}\right) E(x ; \varepsilon) u=f, \quad \lambda>0, \quad 0<\kappa<1,
$$

with $f \in L_{2, \alpha}^{(7)}, 1 / 2<\alpha<(1 / 2) \sigma$. Let $G$ and $\Pi$ be as in (3.8) and (3.18), respectively, and let $\psi(\xi), \chi(\xi)\left(\in A_{0,0}^{(0)}(1)\right)$ and $\omega(\eta)$ be as before. We apply the same argument as in section 3 to (4.1) to obtain the following equation corresponding to (3.21):

$$
\left(D_{t}-A\left(x, D_{y} ; \varepsilon\right)\right) w+S\left(x, D_{x} ; \varepsilon\right) w-i_{\kappa} J K\left(x, D_{x} ; \varepsilon\right)^{2} w=h+q(\varepsilon),
$$


where $w=w(\varepsilon)$ and $h=h(\varepsilon)$ are defined by

$$
\begin{aligned}
& w(\varepsilon)=H\left(x, D_{x} ; \varepsilon\right)\left[\tilde{U}\left(x, D_{x} ; \varepsilon\right) E(x ; \varepsilon)^{1 / 2} \psi\left(D_{x}\right) u\right]^{\wedge}, \\
& h(\varepsilon)=J K\left(x, D_{x} ; \varepsilon\right)\left[\tilde{U}\left(x, D_{x} ; \varepsilon\right) E(x ; \varepsilon)^{-1 / 2} \psi\left(D_{x}\right) f\right]^{\wedge},
\end{aligned}
$$

and $q(\varepsilon)$ satisfies the estimate

$$
|q(\varepsilon)|_{0, \nu+\alpha}^{(4)} \leqq \varepsilon C\left\{|f|_{0, \nu-\alpha}+|u|_{-1, \nu-\alpha}\right\}, \quad 0 \leqq \nu \leqq \alpha,
$$

for $C$ independent of $\varepsilon$. Furthermore, the symbols of pseudo-differential operators in (4.2)-(4.4) have the following properties:

(P.1) Let $a_{ \pm j}(x, \eta ; \varepsilon)\left(=\omega(\eta) \sigma_{ \pm j}(x, \eta ; \varepsilon)\right), 1 \leqq j \leqq 2$, be the (diagonal) elements of diagonal matrix $A(x, \eta ; \varepsilon)$. Then, $a_{ \pm j}(x, \eta ; \varepsilon)$ satisfies the estimate (see (3.19) in Lemma 3.8);

$$
\left|\partial_{x}^{m} \partial_{\eta}^{n} a_{ \pm j}(x, \eta ; \varepsilon)\right| \leqq \varepsilon C(1+|x|)^{-\sigma}(1+|\eta|)^{-|n|}, \quad ' m \mid \geqq 1 .
$$

(P.2) $\varepsilon^{-1} S(x, \xi ; \varepsilon)$ belongs to $A_{\sigma, o}^{(0)}(4)$ uniformly in $\varepsilon$.

(P.3) $K(x, \xi ; \varepsilon), H(x, \xi ; \varepsilon)$ and $\tilde{U}(x, \xi ; \varepsilon)$ take the following form: $K=$ $K^{(0)}(\xi)+K_{r}(x, \xi ; \varepsilon), H=H^{(0)}(\xi)+H_{r}(x, \xi ; \varepsilon)$ and $\tilde{U}=\tilde{U}^{(0)}(\xi)+\tilde{U}_{r}(x, \xi ; \varepsilon)$, where $\varepsilon^{-1} K_{r}$ and $\varepsilon^{-1} H_{r}$ (resp. $\varepsilon^{-1} \tilde{U}_{r}$ ) belong to $A_{\theta, \sigma}^{(0)}(4)$ (resp. $\left.A_{\theta, \sigma}^{(0)}(7)\right)$ uniformly in $\varepsilon$. Furthermore, $K^{(0)}(\xi)$ and $H^{(0)}(\xi)$ are $4 \times 4$ diagonal matrices (see (3.20)) and satisfy the relation $K^{(0)}(\xi) H^{(0)}(\xi)=\chi(\xi)^{2} I_{4}$, and also $\tilde{U}^{(0)}(\xi)$ satisfies $\tilde{U}^{(0)}(\xi)\left(\tilde{U}^{(0)}(\xi)\right)^{*}=\chi(\xi)^{2} I_{7}$.

4.3. The proof of a priori estimate (1.11). For the proof of (1.11), we fix $\varepsilon$ small enough throughout this subsection. (We note that all constants $C$ below may depend on $\varepsilon$.)

First, we introduce several new notations to rewrite the equation (4.2). We write the solution $w$ to equation (4.2) as $w={ }^{t}\left(w_{+1}, w_{+2}, w_{-1}, w_{-2}\right)$ and also use the same notation as above for the terms $h$ and $q(\varepsilon)$ on the right side of (4.2). Next, we set $\tilde{h}={ }^{t}\left(\tilde{h}_{+1}, \tilde{h}_{+2}, \tilde{h}_{-1}, \tilde{h}_{-2}\right)=-S\left(x, D_{x} ; \varepsilon\right) w$. Since $S\left(x, D_{x} ; \varepsilon\right) \in O P A_{\sigma, \sigma}^{(0)}(4)$, we have $|\tilde{h}|_{0, \alpha}^{(4)} \leqq C|w|_{0,-\alpha \cdot}^{(4)}$. Furthermore, for the decomposition $K(x, \xi ; \varepsilon)=K^{(0)}(\xi)+K_{r}(x, \xi ; \varepsilon)$ with $K_{r} \in A_{\theta, o}^{(0)}(4)$ ((P.3)), we denote by $k_{ \pm j}^{(0)}(\xi)\left(\in A_{0,0}^{(0)}(1)\right)$ the (diagonal) elements of diagonal matrix $K^{(0)}(\xi)$, and define $g={ }^{t}\left(g_{+1}, g_{+2}, g_{-1}, g_{-2}\right)$ by $g=-J\left(K\left(x, D_{x} ; \varepsilon\right)^{2}-K^{(0)}\left(D_{x}\right)^{2}\right) w$. Since $K_{r}\left(x, D_{x} ; \varepsilon\right) \in O P A_{\theta, o}^{(0)}(4)$, we have

$$
\kappa\left(|g|_{0, \theta}^{(4)}\right)^{2} \leqq \kappa C\left(|u|_{0,0}\right)^{2} \leqq C|f|_{0, \alpha}|u|_{0,-\alpha} .
$$

With the notations above, we now rewrite the equation (4.2) into the following equivalent form: 
$(4.8)_{ \pm j}$

$$
\partial_{t} w_{ \pm j}-i a_{ \pm j}\left(x, D_{y} ; \varepsilon\right) w_{ \pm j} \pm \kappa k_{ \pm j}^{(0)}\left(D_{x}\right)^{2} w_{ \pm j}=\kappa g_{ \pm j}+p_{ \pm j},
$$

where $p_{ \pm j}=i\left(h_{ \pm j}+\tilde{h}_{ \pm j}+q_{ \pm j}(\varepsilon)\right)$.

Now, we are in a position to apply Propositions 3.2 and 3.3 in [6] to equation $(4.8)_{ \pm j}$.

First, by Proposition 3.2 in [6], we have

$$
\begin{aligned}
& \int_{-\infty}^{\infty}\left(1+t^{2}\right)^{-\nu}\left\|w_{ \pm \jmath}(t)\right\|^{2} d t \\
& \quad \leqq C_{\nu}\left\{\left(\left|p_{ \pm j}\right| \begin{array}{l}
(1), \alpha \\
0, \alpha
\end{array}\right)^{2}+\left(\left|w_{ \pm j}\right| \begin{array}{l}
(1) \\
0,-\alpha
\end{array}\right)^{2}+\kappa\left(\left(\left|w_{ \pm j}\right| \begin{array}{l}
(1) \\
0,0
\end{array}\right)^{2}+\left(\left|g_{ \pm j}\right| \begin{array}{l}
(1) \\
0,0
\end{array}\right)^{2}\right)\right\},
\end{aligned}
$$

for any $\nu, 1 / 2<\nu<\alpha$, where \|\| denotes the norm in $L_{2,0}^{(1)}\left(R_{y}^{2}\right)$. Since $\left|p_{ \pm j}\right|_{0, \alpha}^{(1)} \leqq C\left\{|f|_{0, \alpha}+|u|_{0,-\alpha}\right\}$ and since $\kappa\left(\left|g_{ \pm j}\right|_{0,0}^{(1)}\right)^{2} \leqq C|f|_{0_{0} \alpha}|u|_{0,-\alpha}$, it follows that

$$
|w|_{0,-\nu}^{(4)} \leqq C_{\nu}\left\{|f|_{0, \alpha}+|u|_{0-\alpha}\right\}
$$

for any $\nu, 1 / 2<\nu<\alpha$.

Next, by Proposition 3.3 in [6], we obtain, using the same argument as above, that

$$
\begin{aligned}
& \int_{-\infty}^{-1}\left(1+t^{2}\right)^{(\mu-1) / 2}\left\|w_{+j}(t)\right\|^{2} d t \leqq C\left\{|f|_{0, \alpha}^{2}+|u|_{0,-\alpha}^{2}\right\}, \\
& \int_{1}^{\infty}\left(1+t^{2}\right)^{(\mu-1) / 2}\left\|w_{-j}(t)\right\|^{2} d t \leqq C\left\{|f|_{0, \alpha}^{2}+|u|_{0,-\alpha}^{2}\right\}
\end{aligned}
$$

for any $\mu, 0<\mu<2 \alpha-1$.

Now, we shall prove the a priori estimate (1.11).

THEOREM 4.1. Let $u$ be a solution to equation (3.1) with $f L_{2, \alpha}^{(7)}, 1 / 2<$ $\alpha<(1 / 2) \sigma$. Then, there exists a constant $C_{\nu}$ independent of $\kappa, 0<\kappa<1$, such that for any, $1 / 2<\nu<\alpha$,

$$
|u|_{0,-\nu} \leqq C_{\nu}\left\{|f|_{0, \alpha}+|u|_{0,-\alpha}\right\} \text {. }
$$

Furthermore, the above constant $C_{\nu}$ is taken independently of $\lambda$, if $\lambda$ ranges over a compact interval not containing the origin.

Proof. Let $G$ be as in (3.8). We may assume that $\psi(\xi)=1$ in $G$ by extending $G$ slightly, if necessary. We combine (4.9) with Lemmas 3.6 and 3.7 to obtain

$$
\left.{ }_{1} \psi\left(D_{x}\right) u\right|_{0,-\nu} \leqq C_{\nu}\left\{|f|_{0, \alpha}+|u|_{0,-\alpha}\right\} .
$$

Let $\psi_{r}(\xi)=1-\psi(\xi)$ and define the surfaces $S_{ \pm \jmath}(\lambda)(j=1,2)$ and $S_{p}(\lambda)$ as $S_{ \pm j}(\lambda)=\left\{\xi ; \lambda_{ \pm j}^{(0)}(\xi)=\lambda\right\}$ and $S_{p}(\lambda)=\left\{\xi ; \lambda_{p}^{(0)}(\xi)=\lambda\right\}, \lambda>0$, respectively. 
As is easily seen, the intersection of the support of $\psi_{r}(\xi)$ with $S_{ \pm j}(\lambda)$ and $S_{p}(\lambda)$ is bounded. Therefore, we can apply the same reasoning as in [6] and [7] to equation (4.1') with $\varepsilon$ small enough and obtain

$$
\left|\psi_{r}\left(D_{x}\right) u\right|_{0,-\nu} \leqq C_{\nu}\left\{|f|_{0 \alpha}+|u|_{0,-\alpha}\right\} \text {. }
$$

In particular, the analysis near the double intersecting points of $S_{+1}(\lambda)$ and $S_{+2}(\lambda)\left(S_{-1}(\lambda)\right.$ and $\left.S_{-2}(\lambda)\right)$ uses the idea in [7]. Thus, the desired estimate is obtained and the proof is completed.

\section{§5. The proof of Theorem 1.2}

The proof of Theorem 1.2 is done in almost the same way as in section 8 of [6], and so we give only a sketch here.

Proof of Theorem 1.2. First, we shall prove statement (i) by contradiction. We assume that there exist sequences $\left\{\kappa_{n}\right\}, 0<\kappa_{n}<1$, and $\left\{f_{n}\right\}$, $f_{n} \in L_{2, \alpha}^{(7)}$, such that $\kappa_{n} \rightarrow 0, f_{n} \rightarrow 0$ in $L_{2, \alpha}^{(7)}$ and $\left|u_{n}\right|_{0,-\alpha}=\left|R\left(\lambda+i \kappa_{n}\right) f_{n}\right|_{0,-\alpha}=1$. Then, there exists a subsequence of $\left\{u_{n}\right\}$ (denoted by the same symbol $\left.\left\{u_{n}\right\}\right)$ such that $u_{n} \rightarrow u$ as $n \rightarrow \infty$ weakly in $L_{2,-\alpha}^{(7)}$ Obviously, $u$ satisfies the equation $L u-\lambda u=0$.

We shall show that $u \neq 0$. By Theorem 3.1,

$$
1=\left|u_{n}\right|_{0,-\alpha} \leqq C\left\{\left|f_{n}\right|_{0, \alpha}+\left|u_{n}\right|_{-1,-\alpha}\right\}
$$

for $C$ independent of $n$. Hence, it follows that $\left|u_{n}\right|_{-1,-\alpha} \geqq C$ for $n$ large enough. On the other hand, by Theorem $4.1,\left|u_{n}\right|_{0,-\nu} \leqq C$ for $\nu, 1 / 2<\nu$ $<\alpha$. Since a bounded set in $H_{1, \alpha}^{(7)}$ is pre-compact in $L_{2, \nu}^{(7)},\left\{u_{n}\right\}$ forms a precompact set in $H_{-1,-\alpha}^{(7)}$ by duality. Therefore, $u_{n}$ converges to $u$ strongly in $H_{-1,-\alpha}^{(7)}$ Thus, $u \neq 0$.

Next, we shall show that $u \in L_{2,0}^{(7)}$, which leads us to the contradiction, since $\lambda$ is not an eigenvalue of $L$ by assumption. To prove this, we apply Proposition 3.4 of [6].

We rewrite the equation $L u=\lambda u$ as

$$
L_{0}\left(D_{x}\right) u-\lambda E(x ; \varepsilon) u=\lambda E_{r}(x ; \varepsilon) u
$$

for $\varepsilon$ small enough and reduce (5.1) to an equation of the form (4.2);

$$
\left(D_{t}-A\left(x, D_{Y} ; \varepsilon\right)\right) w+S\left(x, D_{x} ; \varepsilon\right) w=h+q(\varepsilon),
$$

where

$$
\begin{aligned}
& w=w(\varepsilon)=H\left(x, D_{x} ; \varepsilon\right)\left[\tilde{U}\left(x, D_{x} ; \varepsilon\right) E(x ; \varepsilon)^{1 / 2} \psi\left(D_{x}\right) u\right]^{\wedge} \\
& h=h(\varepsilon)=J K\left(x, D_{x} ; \varepsilon\right)\left[\tilde{U}\left(x, D_{x} ; \varepsilon\right) E(x ; \varepsilon)^{-1 / 2} \psi\left(D_{x}\right) E_{r}(x ; \varepsilon) u\right]^{\wedge}
\end{aligned}
$$


and $q(\varepsilon)$ satisfies the estimate (4.5) with $\nu=0$

$$
|q(\varepsilon)|_{0, \alpha}^{(4)} \leqq \varepsilon C|u|_{0,-\alpha}
$$

for $C$ independent of $\varepsilon$. (In the proof of this theorem, we regard $\varepsilon$ as a parameter and denote by $C$ constants independent of $\varepsilon$.)

According to $(4.8)_{ \pm j}$, each component $w_{ \pm j}=w_{ \pm j}(\varepsilon)$ of $w$ obeys the equation

$$
\left(\partial_{t}-i a_{ \pm j}\left(x, D_{y} ; \varepsilon\right)\right) w_{ \pm j}=p_{ \pm j}(\varepsilon) .
$$

where $p_{ \pm j}(\varepsilon)=i\left(h_{ \pm j}+\tilde{h}_{ \pm j}+q_{ \pm j}(\varepsilon)\right)$ and $\tilde{h}=-S\left(x, D_{x} ; \varepsilon\right) w$. Since $\varepsilon^{-1} S\left(x, D_{x} ; \varepsilon\right)$ belongs to $O P A_{\sigma, \sigma}^{(0)}(4)$ uniformly in $\varepsilon((\mathrm{P} .2))$, we have $|\tilde{h}|_{0, \alpha}^{(4)} \leqq \varepsilon C|u|_{0,-\alpha}$.

Now, we want to apply Proposition 3.4 of [6] to equation $(5.4)_{ \pm j}$. To do this, we have to prove that

$$
\liminf _{t \rightarrow \pm \infty}\left\|w_{+j}(t)\right\|^{2}=\liminf _{t \rightarrow \pm \infty}\left\|w_{-j}(t)\right\|^{2}=0,
$$

where \|\| denotes the norm in $L_{2,0}^{(1)}\left(R_{y}^{2}\right)$. However, in view of (4.10), we have only to show that

$$
\liminf _{t \rightarrow \infty}\left\|w_{+j}(t)\right\|^{2}=\liminf _{t \rightarrow-\infty}\left\|w_{-j}(t)\right\|^{2}=0 .
$$

To prove this, we denote by $\langle$,$\rangle the scalar product in L_{2,0}^{(1)}\left(R_{y}^{2}\right)$ and take the scalar product of $w_{+j}$ with equation $(5.4)_{+j}$. Furthermore, we integrate the resulting relation with respect $t$ over $(-T, T)$ and let $T \rightarrow \infty$. Then, taking account of (4.10), we obtain

$$
\lim _{t \rightarrow \infty} \sum_{j=1}^{2}\left\|w_{+j}(t)\right\|^{2}=-2 \sum_{j=1}^{2} \operatorname{Im}\left(h_{+j}, w_{+j}\right)_{1}+O(\varepsilon),
$$

where $(,)_{j}$ denotes the scalar product in $L_{2,0}^{(j)}\left(R_{x}^{3}\right)$. Similarly, taking the scalar product $\langle$,$\rangle of -w_{-j}$ with equation $(5.4)_{-j}$, we obtain

$$
\lim _{t \rightarrow-\infty} \sum_{j=1}^{2}\left\|w_{-j}(t)\right\|^{2}=2 \sum_{j=1}^{2} \operatorname{Im}\left(h_{-j}, w_{-j}\right)_{1}+O(\varepsilon) .
$$

We assert that

$$
\begin{aligned}
& \sum_{j=1}^{2}\left\{\operatorname{Im}\left(h_{-j}, w_{-j}\right)_{1}-\operatorname{Im}\left(h_{+j}, w_{+j}\right)_{1}\right\} \\
& =-\operatorname{Im}\left(\psi\left(D_{x}\right) u, \psi\left(D_{x}\right) E_{r}(x ; \varepsilon) u\right)_{7}+O(\varepsilon) .
\end{aligned}
$$

If we recall the definition (5.3) of $w$ and $h$, this assertion is verified by 
almost the same argument as in section 8 of [6] and hence, using a partition of unity in the $\xi$-space as in [6], we can conclude from $\operatorname{Im}\left(u, E_{r}(x ; \varepsilon) u\right)_{7}$ $=0$ that

$$
\lim _{t \rightarrow \infty}\left\|w_{+j}(t)\right\|^{2}=\lim _{t \rightarrow-\infty}\left\|w_{-j}(t)\right\|^{2}=0 .
$$

Thus, we can prove by Proposition 3.4 of [6] that $w \in L_{2,0}^{(4)}$ and hence it follows immediately that $\psi\left(D_{x}\right) u \in L_{2,0}^{(7)}$.

To see that $\psi_{r}\left(D_{X}\right) u \in L_{2,0}^{(7)}, \psi_{r}(\xi)=1-\psi(\xi)$, we use the same reasoning as in [7] to analysis near the double intersecting points of the surfaces $S_{+1}(\lambda)$ and $S_{+2}(\lambda)\left(S_{-1}(\lambda)\right.$ and $\left.S_{-2}(\lambda)\right)$. Thus, we have $u \in L_{2,0}^{(7)}$ and statement (i) is proved.

The proof of statement (ii) is also done by almost the same argument as in section 8 of [6], and so we omit the detailed proof. In particular, the uniform convergence in $\lambda$ follows from the uniformity in $\lambda$ of a priori estimates (1.10) and (1.11).

\section{§6. The proof of Theorem 1.1}

In this section, we shall prove Theorem 1.1. The proof of this theorem is also done by almost the same way as in section 7 of [6], and so we give only a sketch.

Proof of Theorem 1.1. Let $K$ be a compact interval (fixed arbitrarily) not containing the origin. We have only to show that there exist only a finite number of eigenvalues with finite multiplicity in $K$. We deny this statement and assume that there exist an infinite sequence of eigenvalues $\left\{\lambda_{n}\right\}, \quad \lambda_{n} \in K$, with repetition according to multiplicity; $L u^{(n)}=\lambda_{n} u^{(n)}$, $\left(u^{(n)}, u^{(n)}\right)_{E}=1,(,)_{E}$ being the energy scalar product. Then, there exists a subsequence of $\left\{u^{(n)}\right\}$ (denoted by the same notation $\left\{u^{(n)}\right\}$ ) such that $u^{(n)}$ converges to $u^{(0)}$ weakly in $L_{2,0}^{(7)}$ as $n \rightarrow \infty$. We may assume that $u^{(0)}=0$.

We assert that for some $\mu, \mu>0$, there exists a constant $C_{\mu}$ independent of $n$ such that

$$
\left|u^{(n)}\right|_{0, \mu} \leqq C_{\mu} .
$$

If this assertion is proved, then the proof of the theorem is completed. Indeed, (6.1) implies that $u^{(n)}$ converges to $u^{(0)}$ strongly in $H_{-1,0}^{(7)}$. On the other hand, it follows from Theorem 3.2 that

$$
1=\left(u^{(n)}, u^{(n)}\right)_{E} \leqq C\left|u^{(n)}\right|_{-1,0} .
$$


Therefore, $\left|u^{(0)}\right|_{-1,0} \geqq C$, which contradicts the assumption above.

Now, we shall prove the assertion (6.1) by applying Proposition 3.4 of [6] again. As in the proof of Theorem 1.2, we write the equation $L u^{(n)}$ $=\lambda_{n} u^{(n)}$ as

$$
L_{0}\left(D_{x}\right) u^{(n)}-\lambda_{n} E(x ; \varepsilon) u^{(n)}=\lambda_{n} E_{r}(x ; \varepsilon) u^{(n)}
$$

for $\varepsilon$ small enough, and reduce (6.2) to an equation of the form like $(5.4)_{ \pm j}$;

$$
\partial_{t} w_{j}^{(n)}-i a_{ \pm j}^{(n)}\left(x, D_{y} ; \varepsilon\right) w_{ \pm j}^{(n)}=h_{ \pm j}^{(n)}+\tilde{h}_{ \pm j}^{(n)}+q_{ \pm j}^{(n)}(\varepsilon),
$$

where $w^{(n)}$ (resp. $\left.h^{(n)}\right)$ with components $w_{ \pm j}^{(n)}, j=1,2$, (resp. $h_{ \pm j}^{(n)}$ ) are given as the form like (5.3) with $u=u^{(n)}$ and $\lambda=\lambda_{n}$, while $\tilde{h}^{(n)}=-S^{(n)}\left(x, D_{x} ; \varepsilon\right) w^{(n)}$ and $q^{(n)}(\varepsilon)$ satisfies the estimate (4.5) with $\nu=\alpha$ uniformly in $n$;

$$
\left|q^{(n)}(\varepsilon)\right|_{0,2 \alpha}^{(4)} \leqq \varepsilon C\left|u^{(n)}\right|_{0,0} \leqq \varepsilon C .
$$

We again apply Proposition 3.4 of [6] to equation (6.3) $)_{ \pm j}$ and, for some $\mu, \mu>0$, we obtain

$$
\sum_{j=1}^{2} \int_{-\infty}^{\infty}\left(1+t^{2}\right)^{\mu}\left\|w_{ \pm j}^{(n)}(t)\right\|^{2} d t \leqq C_{\mu}
$$

with $C_{\mu}$ independent of $n$, where \|\| denotes the norm in $L_{2,0}^{(1)}\left(R_{y}^{2}\right)$ again. We derive an estimate similar to (6.4) for three linearly independent directions. If such estimates are obtained, then (6.1) is verified immediately.

Let $z=(t, y), y=\left(y_{1}, y_{2}\right)$, be another orthogonal coordinate system close enough to the original system $x=\left(x_{1}, x_{2}, x_{3}\right)$. (From now on, the notation $z=(t, y)$ is used with the meaning different from the previous one; $t=x_{1}, y=\left(x_{2}, x_{3}\right)$.) We denote by $\zeta=(\tau, \eta), \eta=\left(\eta_{1}, \eta_{2}\right)$, the coordinate system dual to $z$. Then, Lemma 3.8 is still valid for this coordinates, but estimate (3.19) must be modified as follows:

$$
\begin{aligned}
& \left|\partial_{\eta}^{n} \sigma_{ \pm j}(z, \eta)\right| \leqq C_{n}(1+|\eta|)^{1-|n|}, \\
& \left|\partial_{z}^{m} \partial_{\eta}^{n} \sigma_{ \pm j}(z, \eta)\right| \leqq C_{m n}(1+|z|)^{-(1+\delta)}(1+|\eta|)^{1-|n|}, \quad|m| \geqq 1 .
\end{aligned}
$$

Furthermore, for this coordinates, we can derive the following equation of the form similar to $(6.3)_{ \pm j}$ :

$$
\partial_{t} w_{ \pm j}^{(n)}-i \tilde{a}_{ \pm j}^{(n)}\left(z, D_{y} ; \varepsilon\right) w_{ \pm j}^{(n)}=p_{ \pm j}^{(n)}(\varepsilon),
$$

where $p_{ \pm j}^{(n)}(\varepsilon) \in L_{2,2 \alpha}^{(1)}$ and the symbols $\tilde{a}_{ \pm j}^{(n)}(z, \eta ; \varepsilon)$ satisfy the estimates (6.5) uniformly in $n$. Let $\rho_{r}(\eta)=\left(1+\gamma|\eta|^{2}\right)^{-1 / 2}, \gamma, \gamma>0$, being a parameter small enough, and define $\tilde{w}_{ \pm j}^{(n)}$ by $\tilde{w}_{ \pm j}^{(n)}=\rho_{r}\left(D_{y}\right) w_{ \pm j}^{(n)}$. Then, $\tilde{w}_{ \pm j}^{(n)}$ obeys the equation 
$(6.6)_{ \pm j}$

$$
\partial_{t} \tilde{w}_{ \pm j}^{(n)}-i \tilde{a}_{ \pm j}^{(n)}\left(z, D_{y} ; \varepsilon\right) \tilde{w}_{ \pm j}^{(n)}=\tilde{p}_{ \pm j}^{(n)}(\varepsilon),
$$

where $\tilde{p}_{ \pm j}^{(n)}(\varepsilon)=\rho_{\gamma}\left(D_{y}\right) p_{ \pm j}^{(n)}(\varepsilon)+\left[\rho_{\gamma}\left(D_{y}\right), i \tilde{a}_{ \pm j}^{(n)}\left(z, D_{y} ; \varepsilon\right)\right] w_{ \pm j}^{(n)}$. By estimate (6.5), we see that $\left\{\tilde{p}_{ \pm j}^{(n)}(\varepsilon)\right\}$ is a bounded set in $L_{2,2 \alpha}^{(1)}$. We apply Proposition 3.4 of [6] again to equation (6.6) $)_{ \pm j}$ and let $\gamma \rightarrow 0$ to obtain an estimate similar to (6.4). Thus, we have

$$
\left|\psi\left(D_{x}\right) u^{(n)}\right|_{0, \mu} \leqq C_{\mu}
$$

For $C_{\mu}$ independent of $n$. And also we can show by the same reasoning as in section 7 of [6] that

$$
\left|\psi_{r}\left(D_{x}\right) u^{(n)}\right|_{0, \mu} \leqq C_{\mu}, \quad \psi_{r}(\xi)=1-\psi(\xi) .
$$

Therefore, estimate (6.1) is verified and the proof is complete.

\section{§7. Concluding remark}

The assumptions imposed on $E(x)$ in section 1 are rather restrictive and as stated in section 1 , these assumptions are weakened in this section. The statements of Theorems 1.1 and 1.2 are still valid for the following class of perturbations.

Assumption (A').

(A.1') $E(x)$ is positive definite uniformly in $x$ and is decomposed as $E(x)=E_{\ell}(x)+E_{s}(x)$;

(A.2') $\quad E_{\ell}(x)$ satisfies (A.2) and (A.3);

(A.3 $) \quad E_{s}(x)=O\left(|x|^{-(1+\delta)}\right)$ as $|x| \rightarrow \infty$.

If $E(x)$ satisfies (A.3) with $|m|=1$ only, then $E(x)$ can be decomposed into the above form by use of a mollifier technique. Furthermore, if the perturbation $E(x)$ is of short-range class $\left(E_{\ell}(x)=E_{0}\right)$, then any assumption is not required for the derivatives of $E(x)$.

We shall discuss breifly how the proofs of Theorems 1.1 and 1.2 must be modified for the perturbation $E(x)$ satisfying Assumption ( $\left.\mathrm{A}^{\prime}\right)$. The $a$ priori estimate (1.11) is still valid for such a $E(x)$ without any essential change, while the proof for (1.10) has to be modified.

For the decomposition $E(x)=E_{\ell}(x)+E_{s}(x)$, we rewrite the equation (3.1) as

$$
L_{0}\left(D_{x}\right) u-(\lambda+i \kappa) E_{\ell}(x) u=E(x) f+\left(\lambda+i_{\kappa}\right) E_{s}(x) u .
$$

LeMma 7.1. (cf. Lemma 3.1) Let ${ }^{r} \phi(\xi)$ and $\tilde{\Gamma}(\xi)=\phi(\xi) \Gamma(\xi)$ be as in Lemma 3.1. Then, 


$$
\left|\tilde{\Gamma}\left(D_{x}\right) u\right|_{0, \nu} \leqq C_{\nu}\left\{|f|_{0, \nu}+\left|E_{s}(x) u\right|_{-1, \nu}+|u|_{-1,-\alpha}\right\}
$$

for any $\nu,-\alpha \leqq \nu \leqq \alpha$.

The proof is done by applying to equation (7.1) the same argument as in the proof of Lemma 3.1.

Lemma 7.2. (cf. Lemma 3.2) Let $\tilde{\Gamma}_{0}(\xi)=\phi(\xi) \Gamma_{0}(\xi)$ be as in Lemma 3.2. Then, for any $\nu,-\alpha \leqq \nu \leqq \alpha$, we have

$$
\begin{gathered}
\left|\tilde{\Gamma}_{0}\left(D_{x}\right) u\right|_{0, \nu} \leqq C_{\nu}|f|_{0, \nu}, \\
\left|\phi\left(D_{x}\right) u\right|_{0, \nu} \leqq C_{\nu}\left\{|f|_{0, \nu}+\left|E_{s}(x) u\right|_{-1, \nu}+|u|_{-1,-\alpha}\right\} .
\end{gathered}
$$

Proof. Estimate (7.2) follows from the commutativity of $\tilde{\Gamma}_{0}\left(D_{x}\right)$ with $E(x)$ and (7.3) is proved by combining (7.2) with Lemma 7.1.

Let $\psi(\xi)$ and $\tilde{U}(x, \xi)$ be as in subsection 3.2. We note that $\tilde{U}(x, \xi)$ is defined with $E(x)$ replaced by $E_{\ell}(x)$ : similarly for all the symbols below, $H(x, \xi), K(x, \xi), S(x, \xi)$, etc. We define $v$ as

$$
v=\tilde{U}\left(x, D_{x}\right) E_{\ell}(x)^{1 / 2} \psi\left(D_{x}\right) u
$$

for the solution $u$ to equation (7.1) (see (3.11)). As before, We write $v=$ ${ }^{t}\left(v_{0}, v_{p}, v_{n}, \hat{v}\right), \hat{v}={ }^{t}\left(v_{+1}, v_{+2}, v_{-1}, v_{-2}\right)$. Then, the following results are verified by the same argument as in the proof of Lemmas 3.6 and 3.7.

LEMma 7.3. (cf. Lemma 3.6) $v_{p}$ satisfies the estimate

$$
\left|v_{p}\right|(1), C_{0, \nu}^{(1)} \leqq C_{\nu}\left\{\left.f\right|_{0, \nu}+\left|E_{s}(x) u\right|_{-1, \nu}+|u|_{-1,-\alpha}\right\}
$$

for any $\nu,-\alpha \leqq \nu \leqq \alpha$. A similar estimate holds also for $v_{n}$.

LEMMa 7.4. (cf. Lemma 3.7) $v_{0}$ satisfies the estimate

$$
\left|v_{0}\right|(1), \nu \leqq C_{\nu}\left\{|f|_{0, \nu}+|u|_{-1,-\alpha}\right\}
$$

for any $\nu,-\alpha \leqq \nu \leqq \alpha$.

Furthermore, we define $w$ as

$$
w=H\left(x, D_{x}\right)\left[\tilde{U}\left(x, D_{x}\right) E_{\ell}(x)^{1 / 2} \psi\left(D_{x}\right) u\right]^{\wedge}
$$

(see (3.22)). Then, $w$ obeys an equation of the form like (3.21);

$$
\left(D_{t}-A\left(x, D_{y}\right)\right) w+S\left(x, D_{x}\right) w-i_{\kappa} J K\left(x, D_{x}\right)^{2} w=h+q,
$$

where $S\left(x, D_{x}\right) \in O P A_{0, \sigma}^{(0)}(4)$ and $h$ is expressed as $h=h_{1}+h_{2}$, 


$$
\begin{aligned}
& h_{1}=J K\left(x, D_{x}\right)\left[\tilde{U}\left(x, D_{x}\right) E_{\ell}(x)^{-1 / 2} \psi\left(D_{x}\right) E(x) f\right]^{\wedge}, \\
& h_{2}=(\lambda+i \kappa) J K\left(x, D_{x}\right)\left[\tilde{U}\left(x, D_{x}\right) E_{\ell}(x)^{-1 / 2} \psi\left(D_{x}\right) E_{s}(x) u\right]^{\wedge}
\end{aligned}
$$

(see (3.23)), while $q$ satisfies the estimate

$$
|q|_{0, \nu+\alpha}^{(4)} \leqq C_{\nu}\left\{|f|_{0, \nu-\alpha}+\left|E_{s}(x) u\right|_{-1, \nu-\alpha}+|u|_{-1, \nu-\alpha}\right\}
$$

for any $\nu, 0 \leqq \nu \leqq \alpha$, which follows from Lemmas 7.3 and 7.4.

Lemma 7.5. Let $h_{2}$ be as above. Then, there exist two pseudo-diferential operators $B_{j}\left(x, D_{x}\right), j=1,2$, of class $O P A_{0, \sigma}^{(0)}(4)$ by which $h_{2}$ is represented as follows:

$$
h_{2}=B_{1}\left(x, D_{x}\right) \tilde{E}_{s}(x) B_{2}\left(x, D_{x}\right) w+p,
$$

where $\tilde{E}_{s}(x)$ is the $4 \times 4$ diagonal matrix with $(j, j)$-element $3 \leqq j \leqq 7$, of the (diagonal) matrix $E_{s}(x)$ and $p$ satisfies the estimate

$$
|p|_{0, \nu+\alpha}^{(4)} \leqq C_{\nu}\left\{|f|_{0, \nu-\alpha}+\left|E_{s}(x) u\right|_{-1, \nu-\alpha}+|u|_{-1, \nu-\alpha}\right\}
$$

for any $\nu, 0 \leqq \nu \leqq \alpha$.

Proof. First, by the definition of $E(x)$, we may assume that the first three diagonal elements of $E_{s}(x)$ vanish. Let $\phi(\xi)$ be as in Lemma 3.1. We may further assume that there exist a non-negative smooth function $\phi_{0}(\xi)$ with compact support such that $\psi(\varepsilon)+\phi(\xi)+\phi_{0}(\xi)=1$ and we denote by $p$ all terms majorized by the same bound as in (7.7) with another constant $C_{\nu}$. Then, by Lemma 7.2 , we may write

$$
\begin{aligned}
& \tilde{U}\left(x, D_{x}\right) E_{\ell}(x)^{-1 / 2} \psi\left(D_{x}\right) E_{s}(x) u \\
& \quad=\tilde{U}\left(x, D_{x}\right) E_{\ell}(x)^{-1 / 2} \psi\left(D_{x}\right) E_{s}(x) \psi\left(D_{x}\right) u+p,
\end{aligned}
$$

since $\left|E_{s}(x) \phi_{0}\left(D_{x}\right) u\right|_{0, \nu+\alpha} \leqq C_{s}|u|_{-s, \nu-\alpha}$ for any $s$ (large enough). Furthermore, making use of Lemma 3.4, we have

$$
\psi\left(D_{x}\right) u=E_{\ell}(x)^{-1 / 2} \tilde{U}_{*}\left(x, D_{x}\right) v+p .
$$

Therefore, the desired representation (7.6) is obtained by Lemma 3.9 and by Lemmas 7.3 and 7.4 .

The next result corresponds to Theorem 3.1 (a priori estimate (1.10)).

THeOREM 7.1. Assume that the perturbation $E(x)$ satisfies Assumption (A'). Let $u\left(\in L_{2,0}^{(7)}\right)$ be a solution to equation (7.1) with $f \in L_{2, \alpha}^{(7)}, 1 / 2<\alpha<$ (1/2)o. Then, there exists a constant $C$ independent of $\kappa, 0<\kappa<1$, such that

$$
|u|_{0,-\alpha} \leqq C\left\{|f|_{0, \alpha}+\left|E_{s}(x) u\right|_{-1,-\alpha}+|u|_{-1,-\alpha}\right\} \text {. }
$$


Proof. We modify the argument used to derive the estimate (3.28). To do this, we have only to estimate the term

$$
T_{5}=-\operatorname{Im} \int_{-\infty}^{\infty}\left\langle h_{2}, k(t) w\right\rangle d t
$$

where the notations $k(t)\left(=k_{1}(t)^{2}\right)$ and $\langle$,$\rangle are used with the same meanings$ as in subsection 3.4. Since $E_{s}(x)=O\left(|x|^{-(1+\delta)}\right), 2 \alpha<(1+\delta)$, Lemma 7.5 enables us to estimate $T_{5}$ in the same way as $T_{3}$ in subsection 3.4. Thus, we obtain, using (7.7), that

$$
\begin{aligned}
\left|T_{5}\right| & \leqq C_{3} \int_{-\infty}^{\infty}\left(1+t^{2}\right)^{-\alpha}\left\|k_{1}(t) w\right\|^{2} d t \\
& +K_{M}\left\{|f|_{0,-\alpha}+\left|E_{s}(x) u\right|_{-1,-\alpha}+|u|_{-1,-\alpha}\right\}|u|_{0,-\alpha}
\end{aligned}
$$

for $C_{3}$ independent of $M$. The other terms are also estimated in the same way as in subsection 3.4 and (7.8) is proved.

Similarly, we can prove the following result corresponding to Theorem 3.2 .

Theorem 7.2. Assume that $E(x)$ satisfies Assumption (A'). Let $u\left(\in L_{2,0}^{(7)}\right)$ be an eigenfunction of the operator $L$ with eiganvalue $\lambda, \lambda \neq 0$. Then, there exists a constant $C$ such that $|u|_{0,0} \leqq C\left\{\left|E_{s}(x) u\right|_{-1,0}+|u|_{-1,0}\right\}$.

Now, we shall modify the proof of Theorem 1.2 for the perturbation $E(x)$ satisfying Assumption (A').

Modification of the proof of Theorem 1.2. We have only to show that the limit function $u$ of the subsequence $\left\{u_{n}\right\}, u_{n}=R\left(\lambda+i \kappa_{n}\right) f_{n}$, converging weakly in $L_{2,-\alpha}^{(7)}$ does not vanish. This follows from Theorem 7.1 at once. Indeed, by Theorem 7.1, there exists a constant $C$ independent of $n$ large enough such that $\left|E_{s}(x) u_{n}\right|_{-1,-\alpha}+\left|u_{n}\right|_{-1,-\alpha} \geqq C$. Since $\left|u_{n}\right|_{0,-\nu} \leqq C_{\nu}, 1 / 2<\nu$ $<\alpha$, by a priori estimate (1.11) and since $\left|E_{s}(x) u_{n}\right|_{0,0} \leqq C$ by $E_{s}(x)=$ $O\left(|x|^{-(1+\delta)}\right)$, we see that $\left\{u_{n}\right\}$ and $\left\{E_{s}(x) u_{n}\right\}$ form pre-compact sets in $H_{-1,-\alpha}^{(7)}$. Therefore, it follows that

$$
\left|E_{s}(x) u\right|_{-1,-\alpha}+|u|_{-1,-\alpha}>C(>0) .
$$

This implies that $u$ does not vanish.

Similarly, the proof of Theorem 1.1 is modified by use of Theorem 7.2.

\section{Appendix; derivation of equations (1.1) (1.3)}

According to Pai [4] (p. 36), the non-linear system of equations of 
magnetogasdynamics for an ideal plasma (an inviscid, non-heat-conducting and infinitely electrically conducting plasma) is described as follows:

$$
\begin{aligned}
& \nabla \cdot H=0, \\
& \mu_{0}(\partial / \partial t) H+\nabla \times\left(\mu_{0} H \times v\right)=0, \\
& \rho\{(\partial / \partial t) v+(v \cdot \nabla) v\}+\nabla p+\left(\mu_{0} H\right) \times(\nabla \times H)=0, \\
& (\partial / \partial t) \rho+\nabla \cdot(\rho v)=0, \\
& (\partial / \partial t) S+(v \cdot \nabla) S=0, \\
& p=p(\rho, S),
\end{aligned}
$$

where $H$ is the magnetic vector field (3-dimension), $p$ is the pressure, and $v, \rho$ and $S$ are the velocity vector field (3-dimension), density and entropy of the plasma. The first equation in the above system has the character of an initial condition.

We consider a static solution with the density gradient for the above system of equations. For a given constant pressure $p_{0}$, we define $\rho_{0}(x)$ and $S_{0}(x)$ so that

$$
p_{0}=p\left(\rho_{0}(x), S_{0}(x)\right) .
$$

Then, $(H, v, \rho, p, S)=\left(H_{0}, 0, \rho_{0}(x), p_{0}, S_{0}(x)\right), H_{0}$ being a constant magnetic vector field, becomes a static solution to the above system.

To consider the perturbation from the static solution above, we set $H=H_{0}+h, \quad\left(B_{0}=\mu_{0} H_{0}\right), \rho=\rho_{0}(x)+\rho, p=p_{0}+p$, and $S=S_{0}(x)+S$. (For notational convenience, we use the same notations $\rho, p$ and $S$ to denote the perturbed quantities.) Omitting all terms of second order, we obtain the linearized system of equations for $(h, v, \rho, p, S)$;

$$
\begin{aligned}
& \nabla \cdot h=0, \\
& \mu_{0}(\partial / \partial t) h+\nabla \times\left(B_{0} \times v\right)=0, \\
& \rho_{0}(x)(\partial / \partial t) v+\nabla p+B_{0} \times(\nabla \times h)=0, \\
& (\partial / \partial t) \rho+\nabla \cdot\left(\rho_{0}(x) v\right)=0, \\
& (\partial / \partial t) S+(v \cdot \nabla) S_{0}(x)=0, \\
& (\partial / \partial t) p=(\partial p / \partial \rho)_{0}(\partial / \partial t) \rho+(\partial p / \partial S)_{0}(\partial / \partial t) S,
\end{aligned}
$$

where $(\partial p / \partial \rho)_{0}$ and $(\partial p / \partial S)_{0}$ are the values of $(\partial / \partial \rho) p$ and $(\partial / \partial S) p$ at $\left(\rho_{0}(x)\right.$, $S_{0}(x)$ ), respectively. Furthermore, using the relation (A.0), we have

$$
(\partial p / \partial \rho)_{0} \nabla \rho_{0}(x)+(\partial p / \partial S)_{0} \nabla S_{0}(x)=0 .
$$


We insert (A.4) and (A.5) into (A.6) and obtain, using (A.7), the following relation:

$$
(\partial / \partial t) p+(\partial p / \partial \rho)_{0} \rho_{0}(x) \nabla \cdot v=0 .
$$

Thus, if we set $a(x)=(\partial p / \partial \rho)_{0}^{-1} \rho_{0}(x)^{-1}$, then (A.2), (A.3) and (A.8) become the desired equations $(1.1) \sim(1.3)$. Here $c(x)=(\partial p / \partial \rho)_{0}^{1 / 2}$ and $\rho_{0}(x)$ are called the local speed of sound and the equilibrium density, respectively.

In particular, if the given magnetic vector field $H_{0}=0$, then the linearized equation of non-linear system is reduced to the well-known acoustic equation $(\partial / \partial t)^{2} p=c(x)^{2} \rho_{0}(x) \nabla \cdot \rho_{0}(x)^{-1} \nabla p$. The derivation of such an acoustic equation in an inhomogeneous medium can be found in Friedlander's book, "Sound Pulses", (pp. $3 \sim 4$ ), Cambridge University Press, 1958.

\section{REFERENCES}

[1] G. S. S. Avila, Spectral resolution of differential operators associated with symmetric hyperbolic systems, Appl. Anal., 1 (1972), 283-299.

[ 2 ] G. S. S. Avila and D. G. Costa, Asymptotic properties of general symmetric hyperbolic systems, J. Funct. Anal., 35 (1980), 49-63.

[ 3 ] R. Courant and D. Hilbert, Methods of Mathematical Physics, vol. 2, Interscience Publishers, New York, 1962.

[4] S. I. Pai, Magnetogasdynamics and Plasma Dynamics, Springer, New York, 1962.

[5] J. V. Ralston, Local decay of solutions of conservative first order hyperbolic systems in odd dimensional space, Trans. Amer. Math. Soc., 194 (1974), 27-51.

[6] H. Tamura, The principle of limiting absorption for uniformly propagative systems with perturbations of long-range class, Nagoya Math. J., 82 (1981), 141-174.

[ 7 ] - The principle of limiting absorption for propagative systems in crystal optics with perturbations of long-range class, Nagoya Math. J., 84 (1981), 169-193.

[ 8 ] M. Murata, Rate of decay of local energy and wave operators for symmetric systems, J. Math. Soc. Japan, 31 (1979), 451-480.

Department of Applied Physics

Faculty of Engineering

Nagoya University

Furo-cho, Chikusa-ku, Nagoya

464, Japan 